\title{
The equation of state and composition of hot, dense matter in core-collapse supernovae
}

\author{
S. I. Blinnikov ${ }^{1,2,3}$, I. V. Panov ${ }^{2}$, M. A. Rudzsky ${ }^{4}$, and K. Sumiyoshi ${ }^{5,6}$ \\ 1 Max-Planck-Institut für Astrophysik, Karl-Schwarzschild-Strasse 1, Postfach 1523, 85740 Garching, Germany \\ e-mail: seb@mpa-garching.mpg.de \\ 2 Institute for Theoretical and Experimental Physics, B. Cheremushkinskaya St. 25, 117218 Moscow, Russia \\ 3 IPMU, University of Tokyo 5-1-5 Kashiwanoha, Kashiwa, Chiba 277-8568, Japan \\ ${ }^{4}$ Computer Science Department, Technion-Israel Institute of Technology, 32000 Haifa, Israel \\ 5 Numazu College of Technology, Ooka 3600, Numazu, Shizuoka 410-8501, Japan \\ 6 Theory Center, High Energy Accelerator Research Organization (KEK), Oho 1-1, Tsukuba, Ibaraki 305-0801, Japan
}

Received 24 April 2009 / Accepted 16 August 2011

\section{ABSTRACT}

\begin{abstract}
The equation of state (EOS) and composition of matter are calculated for conditions typical for pre-collapse and early collapse stages in core-collapse supernovae. The composition is evaluated under the assumption of nuclear statistical equilibrium, when the matter is considered as an "almost" ideal gas with corrections owing to thermal excitations of nuclei, to free nucleon degeneracy, and to Coulomb and surface-energy corrections. The account of these corrections allows us to obtain the composition for densities that are slightly below the nuclear matter density. Through comparisons with the EOS developed by Shen et al., which is used in most of recent supernova simulations, we examine the differences of our EOS in the multi-composition with the EOS in the approximation of one representative nucleus. We find that widely distributed compositions in the nuclear chart are different because of the different mass formulae we used, while the thermodynamical quantities are quite close to those in Shen's EOS.
\end{abstract}

Key words. nuclear reactions, nucleosynthesis, abundances - stars: neutron - supernovae: general

\section{Introduction}

A proper description of the equation of state (EOS) for subnuclear and supernuclear densities is of vital importance for current studies on the explosion mechanism of core-collapse supernovae (Bethe 1990; Suzuki 1994; Janka et al. 2007). The success of the prompt shock propagation in the supernova mechanism depends on the size of the homologously contracting inner part of a collapsing core. The larger $Y_{\mathrm{e}}$ (the number of electrons per nucleon) at the time of bounce, the smaller the part of matter that will be dissociated and the stronger the prompt shock wave. Therefore, all factors that influence $Y_{\mathrm{e}}$ must be studied thoroughly.

One factor is the mass fraction of the free protons, $X_{\mathrm{p}}$, in the dense matter of the supernova core. Because the rate of electron capture on a free proton is much higher than that on a nucleus, free protons play an important role in establishing the value of $Y_{\mathrm{e}}$ at the neutrino trapping. The abundance of free protons depends sensitively on the nuclear models of dense matter. The variations of $X_{\mathrm{p}}$ amount to more than an order of magnitude in the studies of supernova EOS so far (Cooperstein 1985; Cooperstein \& Baron 1990; Hillebrandt \& Wolf 1985; Hillebrandt 1991; Lattimer et al. 1985; Lattimer \& Swesty 1991; Shen et al. 1998a,b) and can affect the initial strength of shock wave (Bruenn 1989a; Swesty et al. 1994; Sumiyoshi et al. 2004, 2005). As shown by Bruenn (1989a,b), the free proton fraction may differ considerably depending on the nuclear interaction and its model. Another factor is the composition of various nuclei, which exist under the nuclear statistical equilibrium (NSE). The total rate of electron captures on nuclei can dominate that on free protons, if the number of the free protons is small (Hix et al. 2003), and nuclei may affect the dynamics in this way as well. Therefore, it is crucial to evaluate the composition of dense matter in a precise manner.

Another astrophysical problem where an accurate chemical composition is important is the nucleosynthesis of heavy elements. The studies of various models of the explosive nucleosynthesis as well as the rapid neutron capture process (see, e.g. Ptitsyn \& Chechetkin 1982; Woosley \& Hoffman 1992; Nadyozhin et al. 1998; Arnould et al. 2007, and references therein) require knowledge of the chemical composition close to the NSE as an initial condition for nuclear reaction network calculations. Neutron star mergers and collapsar models for gamma-ray bursts may involve similar conditions as well.

The thermodynamic properties of hot, dense matter have been investigated in various approaches: Saha-like equations (Mazurek et al. 1979; El Eid \& Hillebrandt 1980; Murphy 1980; Ishizuka et al. 2003; Nadyozhin \& Yudin 2004), Hartree-Fock approaches (Bonche \& Vautherin 1981; Wolff 1983; Hillebrandt \& Wolf 1985; Lassaut et al. 1987), compressible liquid-drop model (Baym et al. 1971; Lattimer et al. 1985; Lattimer \& Swesty 1991), and the relativistic mean field theory (Sutaria et al. 1999) with local density approximation (Shen et al. 1998a,b). The review of those approaches, beginning from Bethe et al. (1970), can be found in the context of cold neutron star matter in Rüster et al. (2006) and Haensel et al. (2007). In most of the sets of EOS used for recent supernova simulations, the approximation of one species of nuclei (in addition to neutrons, protons and alpha particles) has been adopted (Hillebrandt \& Wolf 1985; Lattimer \& Swesty 1991; Shen et al. 1998b). However, the advance in recent studies of electron capture rates 
on nuclei for a wide mass range (Langanke \& Martínez-Pinedo 2003) necessitates the evaluation of multi-composition of nuclei to determine the total electron capture rate. Although the treatment with one nuclear species should be a good approximation to derive overall thermodynamical properties of dense matter (Burrows \& Lattimer 1984), it is necessary to take into account the multi-composition at high densities $\sim 10^{12} \mathrm{~g} / \mathrm{cm}^{3}$ near the neutrino trapping regime, where nuclear interaction and multicompositional treatment may become more influential in determining the EOS. The description of the EOS with the multicomposition is, of course, crucial to predict the abundance of nuclei and free protons, see e.g. Murphy (1980).

In spite of an extensive use of the Saha equation in the literature, not all relevant physics was included in the published work within this approach from the very beginning. For example, Murphy (1980) has not taken into account the effects of non-ideal nucleon gas. El Eid \& Hillebrandt (1980) have consistently taken into account finite temperature effects in nucleon interactions following El Eid \& Hilf (1977), but they have omitted the Coulomb corrections. Later Hillebrandt \& Müller (1981), Bravo \& García-Senz (1999) and Nadyozhin \& Yudin (2005) included them in different approximations and solved the implicit set of Saha equations. Moreover, Hillebrandt \& Müller (1981) have taken into account an "excluded-volume" effect owing to the finite size of nuclei. It was noted by Hillebrandt (1991) that the number of nuclear species included in the Saha treatment strongly affects the mass fraction of free protons, which may change the effective adiabatic index through electron captures. New papers have appeared after the first submission of our manuscript which discuss many points touched upon below (Hempel \& Schaffner-Bielich 2010; Raduta \& Gulminelli 2010; Odrzywolek 2010).

In this paper we describe our code, which is suitable for studying the properties of dense matter relevant for corecollapsing supernova conditions with an emphasis on the composition of nuclei. Our code is not new: we have already compared our NSE results with the kinetic approach in the paper (Panov et al. 2001), but the code has not been described in detail. Another motivation for our work is the availability of new mass formulae as in Koura et al. (2005) and Möller et al. (1995) and detailed EOS tables such as those given in Shen et al. (1998b). Our aim is to develop and to test a practical and reliable tool for predictions of NSE composition at subnuclear densities.

Following most closely the conventional Saha approach (Clifford \& Tayler 1965) and the method by Mazurek et al. (1979), we extend this approach in the following points:

- At a certain level of approximation we take into account the influence of free nucleon gas on the surface and Coulomb energies of nuclei. We retain some terms that were omitted by Mazurek et al. (1979). However, we do not take into account the "excluded-volume" effect, because we do not pretend to reach very high densities.

- We have an option to include various results for nuclear partition functions like those of Fowler et al. (1978) and more recent partition functions by Engelbrecht \& Engelbrecht (1991) and Engelbrecht et al. (1990).

- Our network is considerably more extensive than the ones used previously. The atomic mass table is updated using recent theoretical compilations of atomic masses. It covers $\sim 20000$ nuclides (Koura 2007, priv. comm.) for the KTUY mass formula (Koura et al. 2005) and 9000 nuclides for the FRDM mass formula (Möller et al. 1995) as an extra option.
To examine the basic properties of the EOS for supernovae in the current formulation, we calculate the properties of dense matter covering a wide range of density, $\rho$, electron fraction, $Y_{\mathrm{e}}$, and temperature, $T$. We report here the results at the equilibrium for the nuclear and electromagnetic processes, i.e. the NSE, for fixed values of $Y_{\mathrm{e}}$ without imposing beta equilibrium. The beta equilibrium is easily incorporated in the Saha approach as in Mazurek et al. (1979) and El Eid \& Hillebrandt (1980), and our code has this option. A brief discussion of the beta equilibrium is also given below. We show the general features of the dense matter (thermodynamical quantities and compositions) for the supernova environment. To assess the dependence on the nuclear mass formulae, we compare several choices. We also examine the similarity to and difference from the single nuclear species treatment by comparisons with the Shen EOS table (Shen et al. 1998b).

We explain the formulation of NSE in Sect. 2.1 with a detailed description of the spin of nuclei in Sect. 2.2. We briefly describe the atomic mass data used for the calculations in Sect. 2.3, the Shen equation of state in Sect. 2.4 and the beta equilibrium in Sect. 2.5. We show the properties of dense matter in the current framework in Sect. 3. The summary and discussion is given in Sect. 4.

\section{Thermodynamics of interacting nuclides}

\subsection{Inclusion of nuclear and Coulomb contributions}

The basis is the equation of NSE with respect to strong and electromagnetic processes for the chemical potentials of nuclei:

$\mu_{i}=Z_{i} \mu_{\mathrm{p}}+\left(A_{i}-Z_{i}\right) \mu_{\mathrm{n}}$.

We denote $i=1=\mathrm{p}$ for proton and $i=2=\mathrm{n}$ for neutron. We use the index $i$ mostly for nuclei $\left(A_{i}, Z_{i}\right)$ with $A>1$, but we include nucleons $(i=1,2)$ in the summation over all species. To find the correct expressions for $\mu_{i}$ we suppose that the free energy of the set of $\left\{N_{i}\right\}$ nuclides is

$F_{\text {nuc }}=\sum_{i} N_{i} \Phi_{i}\left(T,\left\{n_{k}\right\}\right)$.

Here $\Phi_{i}$ is the free energy per nucleus $i$ and it may depend not only on the concentration

$n_{i}=N_{i} / V$

of the $i$ th nucleus, but also on concentrations of some other nuclei $n_{k}$. Then we have by definition

$\mu_{i}=\left.\frac{\partial F_{\mathrm{nuc}}}{\partial N_{i}}\right|_{T, V}=\Phi_{i}+\left.\sum_{k} n_{k} \frac{\partial \Phi_{k}}{\partial n_{i}}\right|_{T}$.

Thus we find for the pressure

$P_{\mathrm{nuc}}=-\left.\frac{\partial F_{\mathrm{nuc}}}{\partial V}\right|_{T,\left\{N_{k}\right\}}=\sum_{k} \mu_{k} n_{k}-F_{\mathrm{nuc}} / V$,

which is consistent with the definition of the grand thermodynamic potential, $P V$. We recall these elementary details here because there are different prescriptions in separating terms in $F_{\text {nuc }}$ in the literature.

For example, let us start with the Coulomb contribution to $F_{\text {nuc }}$. As usual we introduce

$\Gamma_{i}=\frac{1}{k_{\mathrm{B}} T}\left(\frac{e^{2} Z_{i}^{2}}{a_{Z_{i}}}\right)=\frac{e^{2} Z_{i}^{5 / 3}}{k_{\mathrm{B}} T}\left(\frac{4 \pi}{3} n_{\mathrm{e}}\right)^{1 / 3}$, 
with $a_{Z_{i}}$ being the radius of the ion sphere:

$\frac{4 \pi}{3} a_{Z_{i}}^{3}=\frac{Z_{i}}{n_{\mathrm{e}}}$

If $Q_{i}$ denotes the Coulomb correction to the free energy and has the functional form

$Q_{i} \equiv k_{\mathrm{B}} T f_{0}\left(\Gamma_{i}\right)$

then we find the Coulomb contribution to the chemical potential as

$\mu_{i}^{\text {Coul }}=k_{\mathrm{B}} T f_{0}\left(\Gamma_{i}\right)$.

See Sect. 3.4 below for a detailed discussion of nuances in the definition of $\mu_{i}^{\text {Coul }}$.

We closely follow Mazurek et al. (1979) in the assumed form of the free energy, but with some modifications. The main results presented below use the Coulomb correction as in Eq. (8), as did Mazurek et al. (1979). In the end we check the effect of the additional term that appears in Eq. (31) in Sect. 3.4 below. Thus, we put

$\Phi_{\mathrm{p}}=\Phi_{\mathrm{p}}^{(0)}+W+Q_{\mathrm{p}}+B_{\mathrm{p}}$

$\Phi_{\mathrm{n}}=\Phi_{\mathrm{n}}^{(0)}+W+B_{\mathrm{n}}$.

Here $\Phi_{\mathrm{p}}^{(0)}$ and $\Phi_{\mathrm{n}}^{(0)}$ are the Fermi-Dirac expressions for the free energy of non-relativistic nucleons and they are expressed through the Fermi integrals of half-integer index. (The superscript zero indicates that an expression is for non-interacting particles). To calculate the Fermi integrals, we use the code by Nadyozhin (1974a,b) - for an English version see Blinnikov et al. (1996). For the bulk interaction energy of free nucleons we take the zero temperature expression from Mazurek et al. (1979), where they have used the expression of Baym et al. (1971) with minor corrections from Mackie (1976):

$W=W(k, \beta)$

with $k^{3}=1.5 \pi^{2}\left(n_{\mathrm{p}}+n_{\mathrm{n}}\right)$ and $\beta=n_{\mathrm{p}} /\left(n_{\mathrm{p}}+n_{\mathrm{n}}\right)$. We used the same values of parameters entering in Eq. (12) as in formulae (A1-A5) from Mazurek et al. (1979): $k_{0}=1.34 \mathrm{fm}^{-1} ; W_{0}=15.5 \mathrm{MeV}$; $s=27.1 \mathrm{MeV} ; K=268 \mathrm{MeV}$.

For nuclei $(i>2)$ we assume

$\Phi_{i}=\Phi_{i}^{(0)}+Q_{i}+B_{i}$

The binding energy in Eqs. (10), (11) and (13) is

$B_{i}=M_{i}-\frac{A_{i}}{A_{m}} M_{m}$,

with $M_{i}$ being the mass of a nucleus number $i$, which depends on the choice of the reference nucleus $m$. We use ${ }^{56} \mathrm{Fe}$ as the reference nucleus in our calculations. In calculating the total energy we exclude electron mass $m_{\mathrm{e}}$ from the electron energy, then we have

$B_{i}=\Delta M_{i}-\frac{A_{i}}{A_{m}} \Delta M_{m}$,

where $\Delta M_{i}$ is the atomic mass excess, which accounts for the mass of electrons. To compare with Lattimer \& Swesty (1991), one has to take into account that they assume $B_{\mathrm{n}}=0$, where $m=2=n$ in our notation, and $B_{i}=\Delta M_{i}-A_{i} \Delta M_{\mathrm{n}}$.
Considering the Coulomb corrections we follow Lattimer et al. (1985) and take for the total Coulomb energy of a nucleus

$E_{i}^{\text {Coul }}=\frac{3}{5} \frac{Z_{i}^{2} e^{2}}{r_{A}}\left[1-\frac{3}{2} \frac{r_{A}}{a_{Z}}+\frac{1}{2}\left(\frac{r_{A}}{a_{Z}}\right)^{3}\right]$.

Here $r_{A}$ is the nuclear radius, $r_{A}=1.2 \times 10^{-13} A^{1 / 3} \mathrm{~cm}$. Accordingly the Coulomb correction is

$Q_{i}=\frac{3}{5} \frac{Z_{i}^{2} e^{2}}{r_{A}}\left[-\frac{3}{2} \frac{r_{A}}{a_{Z}}+\frac{1}{2}\left(\frac{r_{A}}{a_{Z}}\right)^{3}\right]$.

In the leading term, which is what we need at high density, this is just $-0.9 \Gamma_{i}$ with $\Gamma_{i}$ defined in Eq. (6) when the form (8) is used.

The Boltzmann expression for the chemical potential satisfies the relation

$\exp \left(\mu_{i}^{B} / k_{\mathrm{B}} T\right)=\frac{n_{i}}{\Omega_{i}}\left(\frac{h^{2} N_{0}}{2 \pi k_{\mathrm{B}} T A}\right)^{3 / 2}$.

One has to introduce the corrections $\Delta_{i}$ in addition to $\mu_{i}^{\mathrm{B}}$ :

$\mu_{i}=Z \mu_{\mathrm{p}}^{\mathrm{B}}+(A-Z) \mu_{\mathrm{n}}^{\mathrm{B}}+\Delta_{i}$

where

$\Delta_{i}=\Delta_{i}^{\mathrm{deg}}+\Delta_{i}^{\mathrm{Coul}}+\Delta_{i}^{\mathrm{nuc}}+\Delta_{i}^{\mathrm{sur}}$.

The corrections for degeneracy are

$\Delta_{i}^{\mathrm{deg}}=Z\left(\mu_{\mathrm{p}}^{0}-\mu_{\mathrm{p}}^{\mathrm{B}}\right)+(A-Z)\left(\mu_{\mathrm{n}}^{0}-\mu_{\mathrm{n}}^{\mathrm{B}}\right)$.

Here $\mu^{0}=k_{\mathrm{B}} T \Phi^{0}$ with $\Phi^{0}$ from Eqs. (10), (11), computed via Fermi integrals. For the Coulomb part we have a correction:

$\Delta_{i}^{\text {Coul }}=Z Q_{\mathrm{p}}-Q_{i}$.

For nuclear interactions we take Eq. (26) from Mazurek et al. (1979) which is

$$
\begin{aligned}
\Delta_{i}^{\mathrm{nuc}}= & -B_{i}^{0}+A W+\left(X_{\mathrm{p}}+X_{\mathrm{n}}\right)\left[Z \frac{\partial W}{\partial X_{\mathrm{p}}}+(A-Z) \frac{\partial W}{\partial X_{\mathrm{n}}}\right] \\
& +\left[-\chi_{i}+Z \sum_{A^{\prime}, Z^{\prime}>1} \frac{X_{A^{\prime}, Z^{\prime}}}{A^{\prime}} \cdot \frac{\partial \chi_{A^{\prime}, Z^{\prime}}}{\partial X_{\mathrm{p}}}\right. \\
& \left.+(A-Z) \sum_{A^{\prime}, Z^{\prime}>1} \frac{X_{A^{\prime}, Z^{\prime}}}{A^{\prime}} \cdot \frac{\partial \chi_{A^{\prime}, Z^{\prime}}}{\partial X_{\mathrm{n}}}\right] .
\end{aligned}
$$

Here $B^{0}$ is the binding energy in vacuum and $\chi_{A, Z} \equiv B_{A, Z}-B_{A, Z}^{0}$. This is a direct consequence of the definitions for NSE (1) and for the chemical potential (4). Mazurek et al. (1979) have argued that this long expression can be simplified: "The terms in the last brackets represent the effects of the changes in binding energies of nuclei due to the interactions with the free nucleons... For simplicity we neglect this term in our numerical calculations below". Contrary to them we do not simplify the relation Eq. (23) and take it in full, which requires an additional loop of NSE iterations.

We assume the following correction to the surface energy:

$$
\begin{aligned}
\Delta_{i}^{\text {sur }}= & W_{\text {sur }} A^{2 / 3}\left\{1-\left(1-\frac{W}{W_{\text {nuc }}}\right)^{1 / 2}\right. \\
& \left.\times\left[1-\left(X_{\mathrm{p}}+X_{\mathrm{n}}\right) \frac{\rho}{\rho_{\text {nuc }}}\right]^{4 / 3}\right\} .
\end{aligned}
$$

The expression actually used in the mass formula for the surface energy may be much more complicated than just $W_{\text {sur }} A^{2 / 3}$, but because the factor in braces tends to zero for vanishing $\rho$ this correction does not influence the vacuum values of nuclear masses. 


\subsection{Spin of ground state}

To find the spin of ground states of exotic and experimentally unknown nuclei, we have the simple shell model with the WoodsSaxon potential for defining the scheme of levels and nuclear systematics. It is well known from experiments and calculations using up-to-date versions of the shell model with residual interaction that the spin of ground states of even-even nuclei is equal to zero. The spin of nuclear ground states of odd- $A$ nuclei is determined generally by the spin of the uncoupled nucleon (exceptions: ${ }^{19} \mathrm{~F}_{9},{ }^{23} \mathrm{Na}_{11},{ }^{55} \mathrm{Mn}_{25}$ ). That is why in the present paper we defined the ground-state spin of odd- $A$ nuclei by the spin of an uncoupled proton or neutron according to experimental data and shell model calculations with phenomenological coupling.

The spin of ground state of odd-odd nuclei is defined by the spins of the uncoupled proton, $j_{\mathrm{p}}$, and neutron, $j_{\mathrm{n}}$, and could not be calculated precisely even on the base of the microscopic models of atomic nuclei. That is why we used the simple phenomenological Nordheim rules in the odd-odd case (Nordheim 1950).

For opposite spins of proton and neutron $\left(j_{\mathrm{n}}=l_{\mathrm{n}} \pm 1 / 2, j_{\mathrm{p}}=\right.$ $\left.l_{\mathrm{p}} \mp 1 / 2\right)$ the spin of an odd-odd nucleus is equal to

$I=j_{\mathrm{n}}-j_{\mathrm{p}}$

In the other case $\left(j_{\mathrm{n}}=l_{\mathrm{n}} \pm 1 / 2, j_{\mathrm{p}}=l_{\mathrm{p}} \pm 1 / 2\right)$ it is quite difficult to find the correct value of the ground state spin because it can fall into the wide range

$j_{\mathrm{n}}-j_{\mathrm{p}} \leq I \leq j_{\mathrm{n}}+j_{\mathrm{p}}$

But it is well known from systematics that $I \approx j_{\mathrm{n}}+j_{\mathrm{p}}$ mainly. For simplicity we chose $I=j_{\mathrm{p}}+j_{\mathrm{n}}$.

The value of the ground state spin is unlikely to influence the results of the NSE calculations strongly because in our model the nuclei are mostly in the excited states. We calculated the ground state spin with another approach (Engelbrecht \& Engelbrecht 1991) to check the influence. It is also based on the simple shell model level scheme, and significantly differs from ours only in the case of odd-odd nuclei, for which the spin is defined as $I=\left(j_{\mathrm{p}}+j_{\mathrm{n}}+1\right) / 2$.

We find that the calculated results depend weakly on the different approaches to the ground-state spin calculation only in the region with the significant amount of "heavy" nuclei, where the role of odd-odd nuclei is more important. Because the most abundant nuclei are even-even and odd- $A$ ones under NSE conditions, one can use any simple estimation of the ground state spin of nuclei under these conditions. For $T_{9}<10$ and low $\rho$ one should prefer our model of the ground-state spin evaluation or an "exact" calculations of nuclear characteristics from first principles.

We agree with the conclusions of Liu et al. (2007) about the role of the excited states, the effect is not very large. Liu and collaborators used a reliable method of the nuclear partition function calculation based on the Fermi gas formula. However, the question deserves further investigation, especially considering the new data from Rauscher et al. (1997); Rauscher \& Thielemann (2000); Rauscher (2003). The expression used in Liu et al. (2007) is related to an energy-dependent level density parameter with microscopic correction to a nuclear mass model (Rauscher et al. 1997; Rauscher \& Thielemann 2000; Rauscher 2003). Our code can incorporate various options for the partition functions.

\subsection{Nuclear masses}

We adopt the table of nuclear masses (KTUY) by Koura et al. (2005) for our EOS calculations. The prediction of nuclear masses by Koura et al. (2005) is based on the mass formula composed of macroscopic and microscopic terms (Koura et al. 2000), which treat deformation, shell and even-odd energies. It covers $\sim 9000$ species of nuclei $(Z \geq 2$ and $N \geq 2)$ in the nuclear chart. We use the extended mass table containing 20000 species (Koura 2007, priv. comm.) to cover the neutron- and proton-rich regions in the NSE. The root-mean-square deviation from experimental masses is $666.7 \mathrm{keV}$. We examine the dependence on the nuclear mass by adopting other tables of the nuclear masses by Hilf et al. (1976). The mass data by Möller et al. (1995) will be implemented in the code. Those mass tables predict the experimental masses very well around the stability line in the nuclear chart, however, they provide different predictions in neutron-rich region away from the stability. It would be interesting to examine whether these differences appear in the composition of dense matter in supernova cores.

\subsection{Shen equation of state}

We adopt the table of EOS by Shen et al. (1998a,b) to investigate the influence of mixture of nuclei on the equation of state. The Shen EOS is a set of thermodynamical quantities of dense matter for the wide range of density, proton fraction and temperature for supernovae. It has been widely used for numerical simulations of core-collapse supernovae (Sumiyoshi et al. 2005, 2006; Janka et al. 2005; Burrows et al. 2006) and other astrophysical phenomena (Rosswog \& Liebendörfer 2003). The equation of state is calculated by the relativistic mean-field theory (Serot \& Walecka 1986), which is based on the relativistic Brückner-Hartree-Fock theory (Brockmann \& Machleidt 1990) and is constrained by the experimental data on neutron-rich nuclei (Sugahara \& Toki 1994). The nuclei in dense matter are described within the local density approximation assuming one species of nucleus surrounded by neutrons, protons, and alpha particles in the Wigner-Seitz cell. The basic behaviour of the Shen EOS in supernova core as well as the comparison with another EOS by Lattimer \& Swesty (1991) can be found in Sumiyoshi et al. (2004, 2005).

\subsection{Beta equilibrium}

Although we fix the value of $Y_{\mathrm{e}}$ in the current calculations, we can impose the condition of beta equilibrium in the Saha approach. We add the formulation here for the applications to proto-neutron stars.

The condition of beta equilibrium is governed by the relation

$\mu_{\mathrm{p}}+\mu_{\mathrm{e}}=\mu_{\mathrm{n}}+\mu_{v}$

Therefore, one has to calculate chemical potentials of neutrinos and electrons in an additional loop of iterations.

For calculating lepton chemical potentials one can use simple relations derived by Nadyozhin; Nadyozhin (1974a,b; see also Blinnikov 1987; Blinnikov \& Rudzsky 1988):

$n-\tilde{n}=\frac{\partial P}{\partial \mu} \mid T=\frac{g}{6 \pi^{2}}\left(\mu^{3}+\pi^{2} \mu T\right)$.

Here $n$ is a fermion number in the unit volume, i.e., the fermion concentration, $\tilde{n}$ is an anti-fermion concentration. $n-\tilde{n}$ is a charge of the unit volume, for example, for the electrons and 

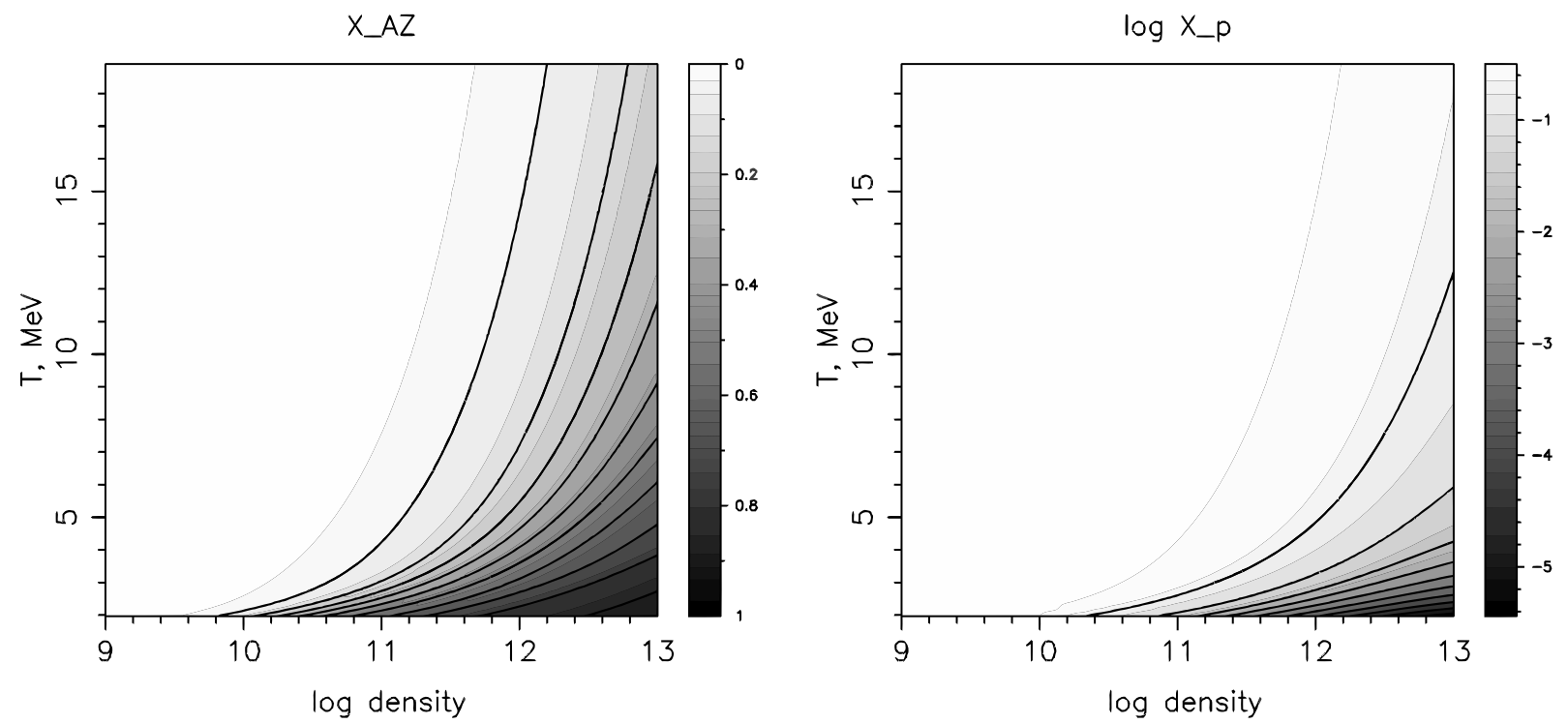

Fig. 1. Isocontours of the mass fraction of heavy nuclei, $X_{\mathrm{AZ}}$, (left $)$ and free protons, $X_{\mathrm{p}},($ right $)$ for $Y_{\mathrm{e}}=0.316$ are shown in the plane of density and temperature.

it is a lepton charge for neutrinos ( $g=2$ for electrons and $g=1$ for neutrinos). It is possible to express the lepton chemical potentials $\mu$ through the radicals if $n-\tilde{n}$ and $T$ are given. In actual calculations it is faster to do this by Newton iterations.

\section{Results}

We report the numerical results in a set of selected conditions for density, $\rho$, electron fraction, $Y_{\mathrm{e}}$, and temperature, $T$. We show the properties of dense matter at typical conditions $\left(\rho=10^{10}\right.$ $10^{13} \mathrm{~g} / \mathrm{cm}^{3}$ ) during the core collapse, where the composition of nuclei and free protons are crucial to determine the electron capture. We remark that the present framework is generally applicable in the wide range of conditions that are necessary for core-collapse supernova simulations. However, it is limited from below by $Y_{\mathrm{e}} \sim 0.3$ because of the coverage of nuclear mass models at neutron-rich region. The limited range of low $T \sim 2 \mathrm{MeV}$ and high $\rho \sim 10^{13} \mathrm{~g} / \mathrm{cm}^{3}$ is because we consider the nuclear interaction and the convergence of iterations.

We show at first the general behaviour of equation of state in the current framework with the mass formula by Koura et al. (2005) in Sect. 3.1. Next, we compare the equation of state obtained by the current Saha-approach with that of Shen EOS (Shen et al. 1998b) in Sect. 3.2. We examine the difference between the two nuclear mass models. We also compare this with the results calculated in the formulation of Mazurek et al. (1979). In Sect. 3.3 we show the composition of nuclei in the nuclear chart for supernova cores.

\subsection{General behaviour}

We show in Figs. 1-4 the general features of dense matter at $Y_{\mathrm{e}}=0.316$ for a wide range of density and temperature relevant to collapsing supernova cores. We also calculated the cases with $Y_{\mathrm{e}}=0.398$ and $Y_{\mathrm{e}}=0.473$, which are not shown in Figs. 1-4, but they are partly discussed below. The values of $Y_{\mathrm{e}}$ are selected to match the values in the original table of Shen EOS. We see very smooth variations of the calculated quantities in the range of density and temperature shown in the plot. There are some wiggles in the plots of the average of mass number, $A$, and proton number, $Z$ in Figs. 2 and 3 because of the shell effects. One can recognize the appearance of nuclei at densities $\sim 10^{10} \mathrm{~g} / \mathrm{cm}^{3}$ at the lowest temperature. The appearance of nuclei occurs with the composition, which is different from that in the Shen EOS, as we will see in the next subsection.

\subsection{Comparisons among models}

We compare the current results with those in the Shen EOS to see the effect of multi-composition with respect to the one-species treatment, which has been used in most of the supernova EOS's. We also examine the dependence on the nuclear mass models by comparing the cases by Koura et al. (2005) and Hilf et al. (1976). We set here $Y_{\mathrm{e}}=0.316$ and $T=2 \mathrm{MeV}$, which roughly corresponds to the neutron-rich supernova core. This $T$ value also corresponds to the lower $T$ boundary in the figures shown above.

We show the mass fractions of nuclei, free protons, and ${ }^{4} \mathrm{He}$ (alpha-particles) as a function of density in Figs. 5 and 6 . As the density increases, we found that the light nuclei other than ${ }^{4} \mathrm{He}$ appear in the current approach. This behaviour takes place because we treat the multi-composition including light nuclei other than ${ }^{4} \mathrm{He}$ in addition to alpha-particles. In the Shen EOS, on the other hand, alpha-particles appear in a larger amount in the density region $\sim 10^{11} \mathrm{~g} / \mathrm{cm}^{3}$, as can be seen in Fig. 6. Figure 7 elucidates the differences of composition. From $\sim 10^{9} \mathrm{~g} / \mathrm{cm}^{3}$, the Saha-treatment provides nuclei of average mass number $A \sim 10$. At densities higher than $\sim 3 \times 10^{11} \mathrm{~g} / \mathrm{cm}^{3}$, the average mass number is smaller than that of representative nuclei in the Shen EOS. We note that the average mass number in the Saha-treatment does not include the contribution of alpha particles, which are treated separately in the Shen EOS.

The appearance of light clusters has been studied in microscopic approaches (for example, Röpke et al. 1982, 1983; Typel et al. 2010) and was recently applied to astrophysics. The mixture of light elements has been demonstrated in the profiles of supernova cores by O'Connor et al. (2007); Sumiyoshi \& Röpke (2008); Arcones et al. (2008). Our findings in the current study 
A average

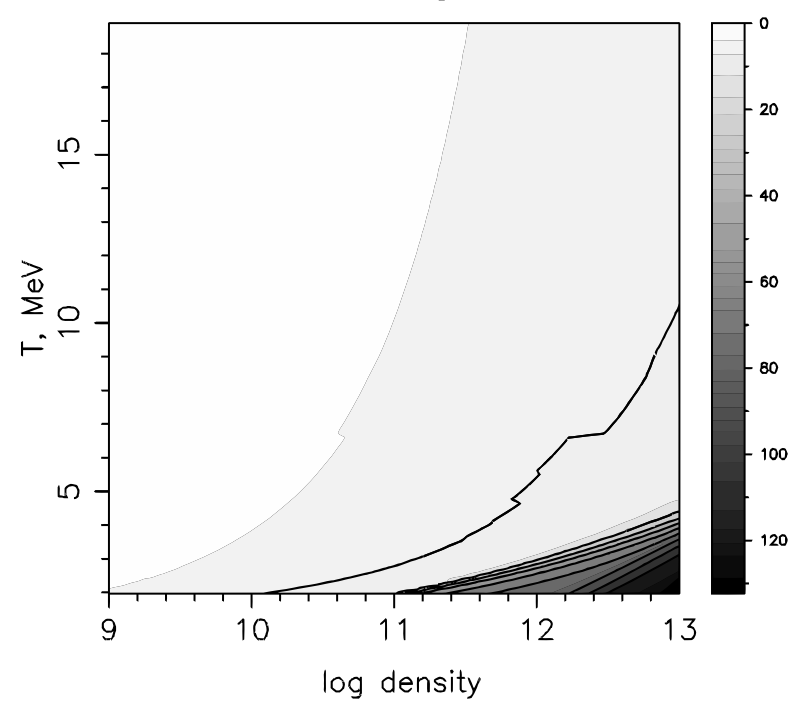

A average

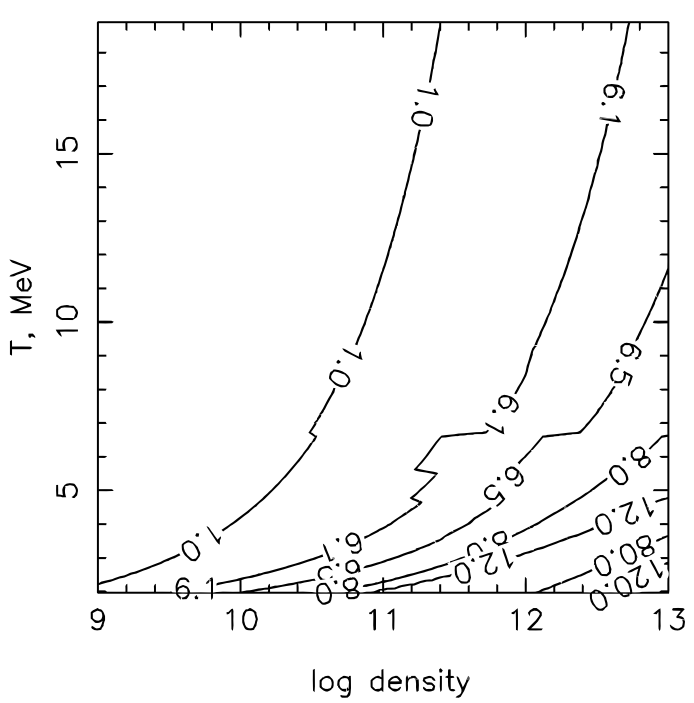

Fig. 2. Isocontours of the average mass number, $A$, of heavy nuclei, for $Y_{\mathrm{e}}=0.316$.
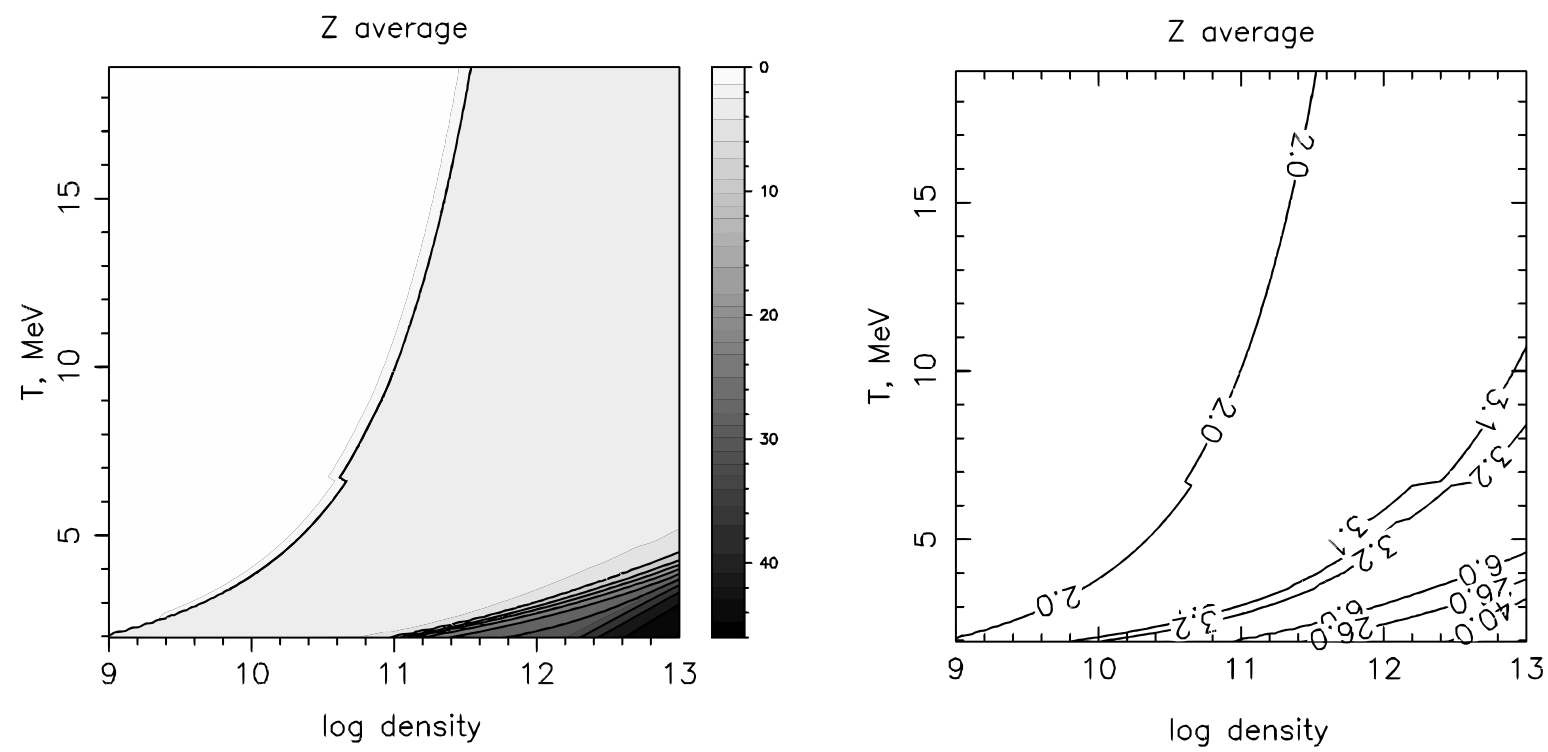

Fig. 3. Isocontours of the average proton number, $Z$, of heavy nuclei, for $Y_{\mathrm{e}}=0.316$.

agree with those recent results, but one has to remember that we have obtained them long ago, because our numerical code has been presented already in Panov et al. (2001). The appearance of light elements may influence the average reaction rate of neutrinos on nuclei, resulting in possible modifications (by factors) of the rates of scattering, emission, and absorption through nuclear structures different from nucleons or ${ }^{4} \mathrm{He}$. It is necessary to evaluate the mean free path using the sum of individual crosssections weighted by the number density of corresponding target nuclei (see, e.g., Arcones et al. 2008; Nakamura et al. 2009; Odrzywolek 2009; Furusawa et al. 2011). Because the crosssections for the coherent scattering on nuclei is $\propto A_{i}^{2}$ the result is quite different when only alpha-particles represent the whole set of light nuclei. For the given density the number of nuclei is $\propto A^{-1}$, so the extinction coefficient goes as $A^{2} / A \propto A$, and the neutrino mean free path $\propto A^{-1}$. This has a substantial effect on the mean free path, e.g. for the conditions in Figs. 6 and 7 when in the realistic calculation the mass fraction of light nuclei with $A_{i}>4$ at $\rho \sim 10^{11} \mathrm{~g} / \mathrm{cm}^{3}$ is larger than that of ${ }^{4} \mathrm{He}$.

The thermodynamical quantities, i.e. entropy and pressure of nuclear contribution, are shown in Fig. 8. The entropy of the Shen EOS agrees very well with the current result. The pressure of the Shen EOS has a trough around $10^{13} \mathrm{~g} / \mathrm{cm}^{3}$ because of the decrease of nuclear part of free energy. As already reported in Shen et al. (1998a), this is caused by the increasing binding energy of nuclei. The occurrence of the drop of nuclear pressure depends on the temperature and electron fraction. Because the pressure consists of contributions from the thermal part and the nuclear interactions, the nuclear pressure of the mixture of nucleons and nuclei is determined by a delicate sum. (We point out that the negative Coulomb corrections are not included into our plots for $P_{\text {nuc }}$, see the discussion in Sect. 3.4.) The pressure also depends on the treatment of the transition from the mixture of nuclei to the uniform matter (which we do not touch upon in 

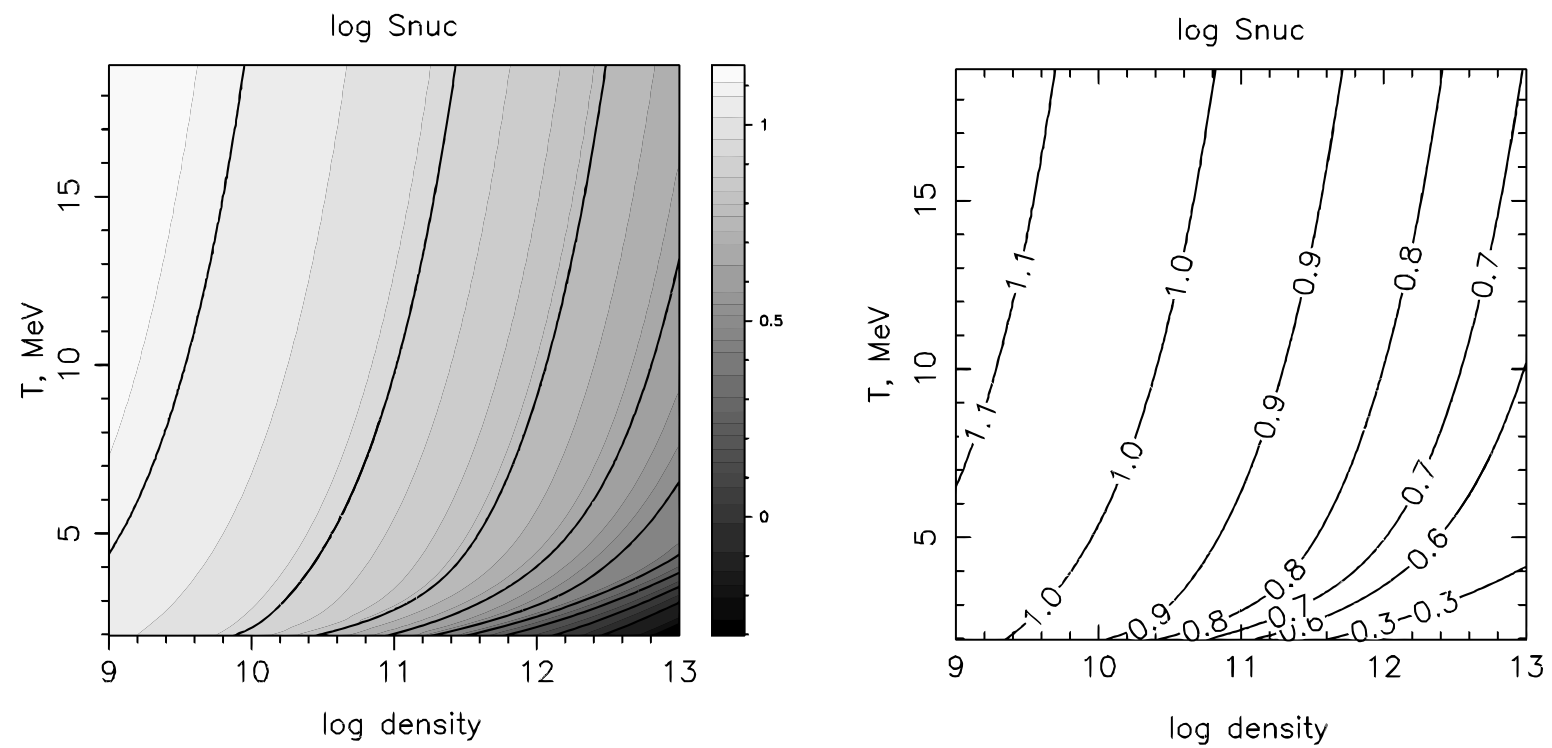

Fig. 4. Isocontours of the nuclear contribution to entropy, $S_{\text {nuc }}$, for $Y_{\mathrm{e}}=0.316$ in the plane of density and temperature.
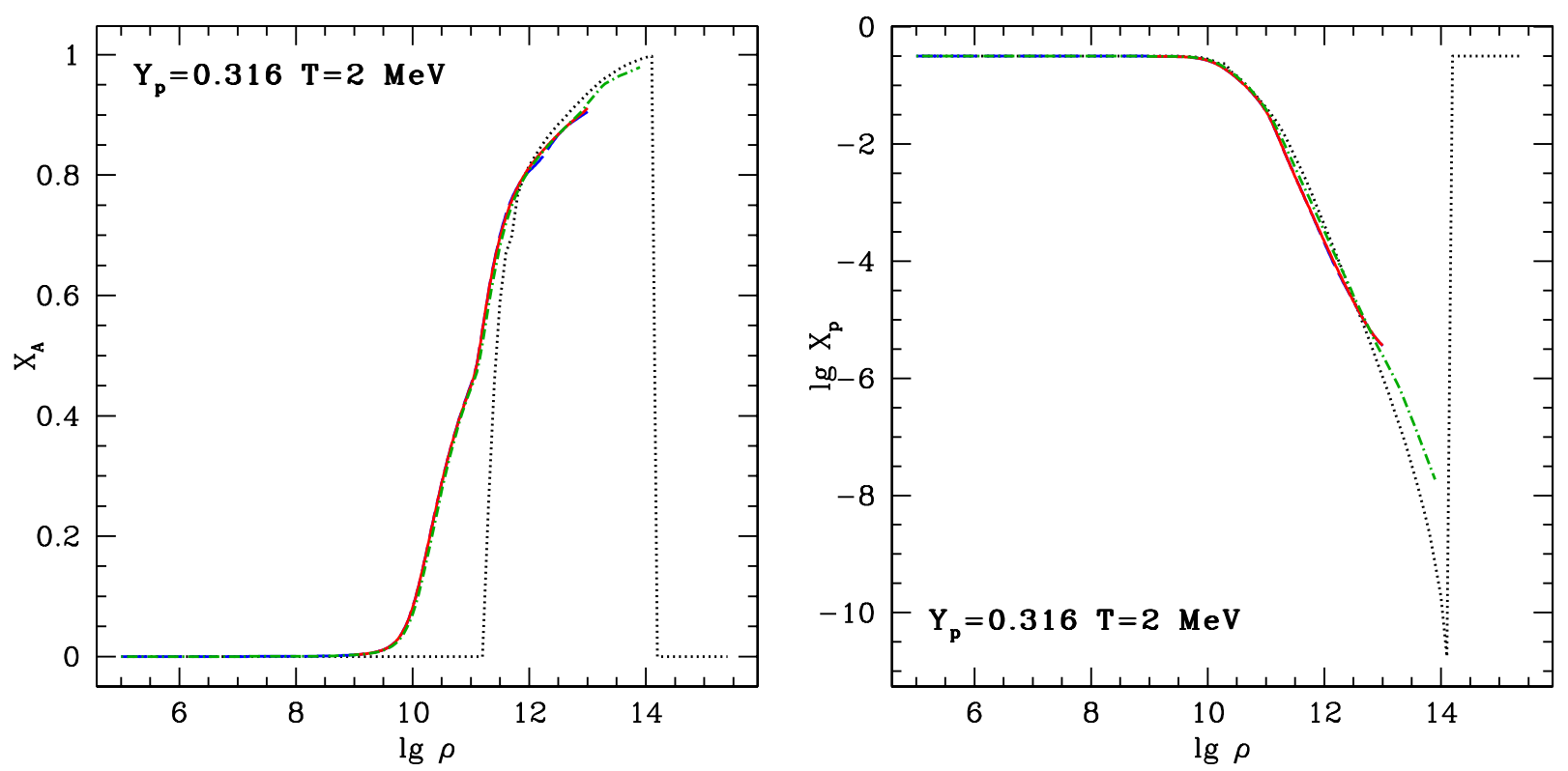

Fig. 5. Mass fraction of nuclei, $X_{A}$, (left $)$ and free protons, $X_{\mathrm{p}}$, (right) as a function of density for $Y_{\mathrm{e}}=0.316$ and $T=2 \mathrm{MeV}$. The solid and dashed lines denote the NSE calculation with mass-formulae by (Koura 2007, priv. comm.) and Hilf et al. (1976), respectively. The dash-dotted lines are obtained using (Koura 2007, priv. comm.), but with a simplified treatment of relation Eq. (23) as in Mazurek et al. (1979). The dotted lines are the results from Shen et al. (1998b).

the current work). This question is analyzed in a recent paper by Hempel \& Schaffner-Bielich (2010), who obtain a good agreement with negative $P_{\text {nuc }}$ in Shen et al. (1998a) when they use the Maxwell-construction in phase transition to uniform nuclear matter. They find that the use of a Gibbs-construction would result in a strictly increasing pressure. In Fig. 22 of Raduta \& Gulminelli (2010), the baryonic pressure stays positive and does not show any drop. Raduta \& Gulminelli (2010) analysed in detail the difference of this property with single nucleus treatments like Lattimer-Swesty EOS (Lattimer \& Swesty 1991). Our NSE approach is less sophisticated than that of Raduta \& Gulminelli (2010), but of course it is much closer to them than to Lattimer \& Swesty (1991); Shen et al. (1998b). We note that one should add lepton and radiation contributions, therefore, the total pressure is always positive, even considering negative Coulomb corrections (see also Fig. 23 in Raduta \& Gulminelli 2010).

In Fig. 9 we show the average mass number and proton number of nuclei for $Y_{\mathrm{e}}=0.473$ and $T=0.63 \mathrm{MeV}$, which is closer to the condition at the centre of the initial progenitors. We can see that the mass number (proton number also) in the current treatment is larger at low density and smaller at high density. This is quite similar to the more neutron-rich and higher temperature case of $Y_{\mathrm{e}}=0.316$ and $T=2 \mathrm{MeV}$ in Fig. 7 .

Now let us comment on the difference in the calculated EOS if we use the treatment of by Eq. (23) suggested by Mazurek et al. (1979). The results of calculations made without the second line in Eq. (23), which expresses the dependence of binding energy of nuclei on the nucleon fractions, are shown by the 


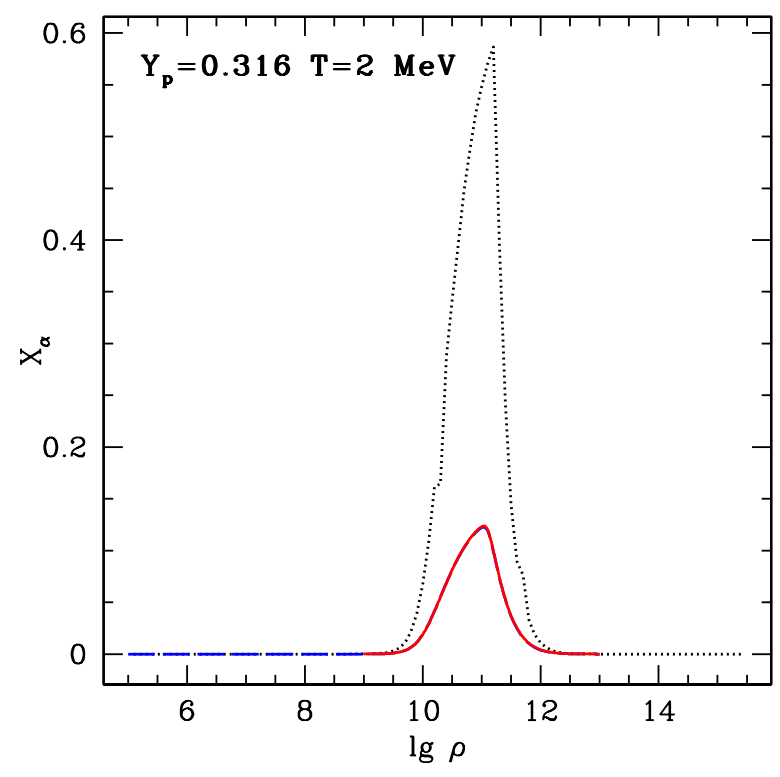

Fig. 6. Mass fraction of alpha-particles, $X_{\alpha}$, as a function of density for $Y_{\mathrm{e}}=0.316$ and $T=2 \mathrm{MeV}$. The notation is the same as in Fig. 5 .

dash-dotted lines in Figs. 5 to 8. In general, the treatment without iterations gives similar results to the one with the iterations to calculate consistently the modified binding energy owing to the presence of nucleons at this condition. In Fig. 10 we show the mass fraction of free protons as a function of density for $Y_{\mathrm{e}}=0.398$ and $T=2 \mathrm{MeV}$. We found that the approximation used by Mazurek et al. (1979) for Eq. (23) gives similar results to those of the Shen EOS and our treatment yields a cosiderably lower proton fraction. This may influence the electron capture rate in collapsing supernova cores. It is worth exploring the reasons for the difference and its dependence on $Y_{\mathrm{e}}$, but this is better conducted with a more modern approach to NSE as in Hempel \& Schaffner-Bielich (2010); Raduta \& Gulminelli (2010), which is beyond the scope of the current paper.

We also explored the dependence of the bulk thermodynamics on the nuclear mass models. The calculations by the two mass models used by us are similar to each other and there is hardly any difference. Only in the prediction of mass number and proton number, the models differ slightly. In this respect the derivation of bulk quantities such as entropy and pressure, can safely be calculated irrespective of the uncertainties of mass formulae. We note, however, that the detailed composition depends on the adopted mass formula, as we will show below.

\subsection{Nuclear composition}

We explore the calculated composition using the mass formula by Koura et al. (2005) in Figs. 11-13 for three typical conditions of the supernova core. We took the conditions from the numerical simulation of core-collapse from a $15 M_{\odot}$ star by Sumiyoshi et al. (2005).

At $\rho=10^{11} \mathrm{~g} / \mathrm{cm}^{3}$, where electron captures continue during the gravitational collapse from the initial Fe core, nuclei up to $A \sim 100$ appear mostly with the peak abundance at $Z \sim 34$ and the magic number $N=50$. By the time the density reaches $\rho=10^{12} \mathrm{~g} / \mathrm{cm}^{3}$, electron capture is about to cease and $Y_{\mathrm{e}}$ becomes smaller. Because of the higher temperature owing to the collapse, the distribution becomes wider, reaching $A \sim 132$ at the double magic nuclei, ${ }^{132} \mathrm{Sn}$. Neutron-richness (small $Y_{\mathrm{e}}$ ) makes the peak position at more neutron-rich, but at $N=50$. At high density of $10^{13} \mathrm{~g} / \mathrm{cm}^{3}$ and high temperature of $3 \mathrm{MeV}$, which corresponds to the moment just before the core bounce, the distribution extends from nucleons to $A \sim 160$ continuously. We see the effect of magic numbers for neutron number 50 and 82 and even-odd numbers, which exist in nuclear mass data.

To demonstrate the difference of composition due to the mass models, we show the case of $\rho=10^{12} \mathrm{~g} / \mathrm{cm}^{3}$ with the mass data by Hilf et al. (1976) in Fig. 14. Through the comparison with Fig. 12, the distribution is narrower both in $N$ and $A=N+Z$ directions. The peak positions are quite close to each other in the two models. We see similar differences also in the case of $\rho=10^{11} \mathrm{~g} / \mathrm{cm}^{3}$ and $\rho=10^{13} \mathrm{~g} / \mathrm{cm}^{3}$, especially on the strength of magic numbers. Neutron-richness at high density causes more pronounced differences in composition among them.

\subsection{Difference in Coulomb corrections}

In this section we briefly discuss the different prescriptions on the form of Coulomb corrections and their influence on the composition of hot, dense matter. There are several expressions of the form of Coulomb corrections found in the literature. These expressions have been used for astrophysics as well as material science and are still under debate. Allmost all of them use a form for the Coulomb free energy like in Eq. (8). However, the expression for $\Gamma_{i}$ may be understood differently. The most widely used expression is Eq. (9) based on $\Gamma_{i}$ from Eq. (6). The question is how to treat the electron number density $n_{\mathrm{e}}$ that defines the radius of the ion sphere in (7).

The standard approach is to forget about the dependence of $n_{\mathrm{e}}$ in (7) on ion numbers and to treat $n_{\mathrm{e}}$ as an independent variable. Then we obtain the chemical potential for ions (9). The other approach substitutes $n_{\mathrm{e}}=\sum_{i} Z_{i} n_{i}$ from the electroneutrality into the expression for the free energy (8), which leads to another expression for the chemical potential that we will discuss below. The assumptions are different, however, their influence on supernova matter has not been studied so far.

We briefly illustrate the latter approach here in the simplest case of a fully ionized plasma with only one ion $\left(A_{i}, Z_{i}\right)$. Then we have $n_{\mathrm{e}}=Z_{i} n_{i}$, and instead of (6) we may define $a_{Z_{i}}$ as

$\frac{4 \pi}{3} a_{Z_{i}}^{3}=n_{i}^{-1}$,

and then we write

$\Gamma_{i}=\frac{1}{k_{\mathrm{B}} T}\left(\frac{e^{2} Z_{i}^{2}}{a_{Z_{i}}}\right)=\frac{e^{2} Z_{i}^{2}}{k_{\mathrm{B}} T}\left(\frac{4 \pi}{3} n_{i}\right)^{1 / 3}$

and obtain

$\mu_{i}^{\text {Coul }}=k_{\mathrm{B}} T\left[f_{0}\left(\Gamma_{i}\right)+\frac{1}{3} \frac{\partial f_{0}}{\partial \ln \Gamma_{i}}\right]$

contrary to our Eq. (9), to Eq. (17) of Mazurek et al. (1979), and to Eq. (97) of Yakovlev \& Shalybkov (1989). The expression $\mu_{i}=k_{\mathrm{B}} T f_{0}\left(\Gamma_{i}\right)$ is standard, but it leads to zero correction for $P_{\text {nuc }}$ in Eq. (5). On the other hand, the expression Eq. (31) gives the expected correction to $P_{\text {nuc }}$ after substitution into Eq. (5).

Basically, the formulae in Yakovlev \& Shalybkov (1989) are correct, because one is free to separate the Coulomb contributions and ascribe them either to electrons or ions, but one has to avoid confusion with this separation. Haensel et al. (2007; Chabrier \& Potekhin see also 1998) rely mostly on the free energy $F$ instead of $\mu_{i}$ and avoid any confusion. Indeed, the expression that is equivalent to (31) appeared already in Dewitt et al. (1973). As we noticed after Eq. (17), we have in the leading term 

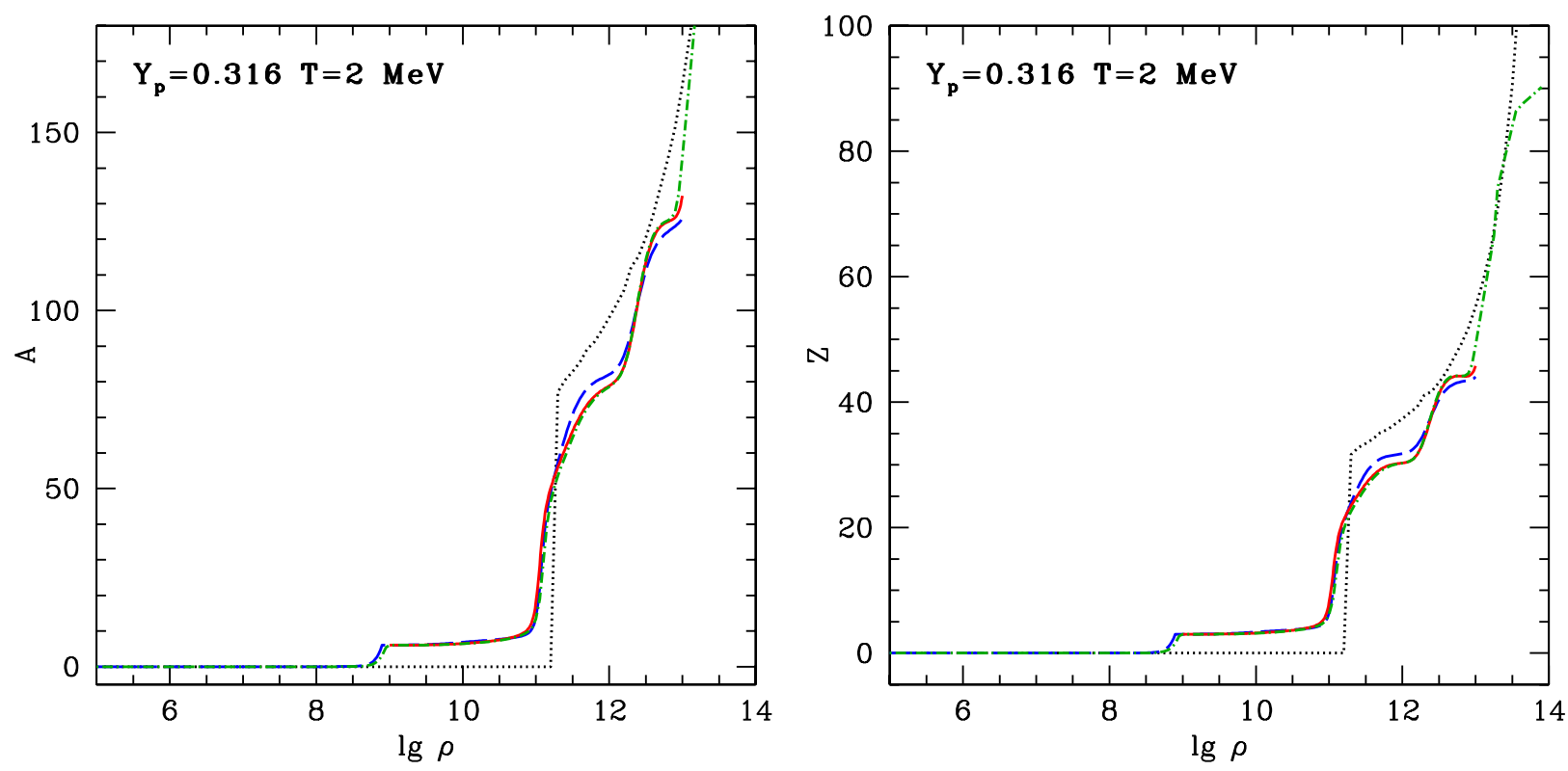

Fig. 7. Average mass number, $A$, (left $)$ and average proton number, $Z$, (right) of nuclei as a function of density for $Y_{\mathrm{e}}=0.316$ and $T=2 \mathrm{MeV}$. The notation is the same as in Fig. 5.
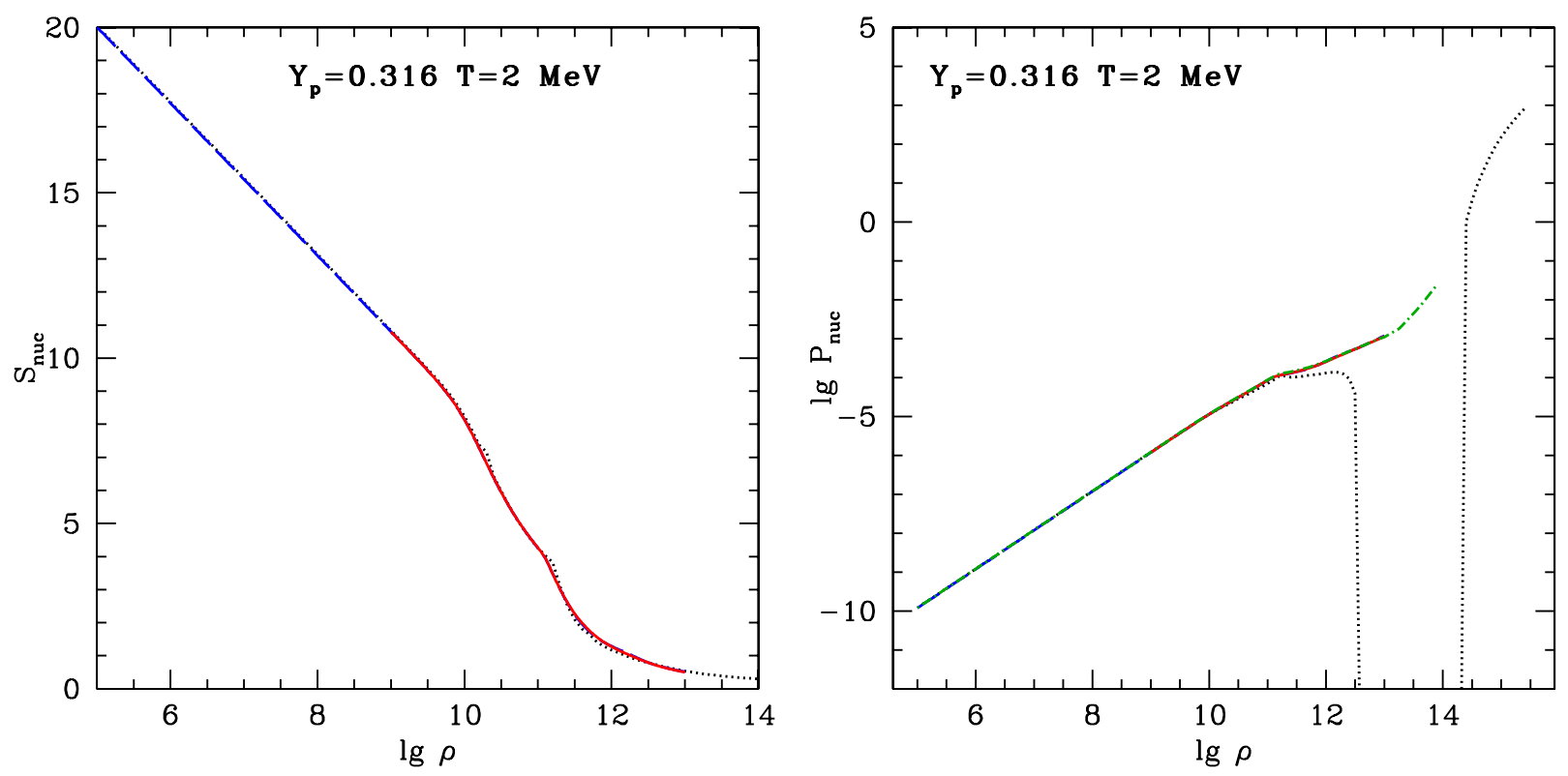

Fig. 8. Entropy, $S_{\text {nuc }}(l e f t)$, and pressure $P_{\text {nuc }}($ right $)$, of nuclei as functions of density for $Y_{\mathrm{e}}=0.316$ and $T=2 \mathrm{MeV}$. The notation is the same as in Fig. 5.

at high density just $-0.9 \Gamma_{i}$ with $\Gamma_{i}$ defined in Eq. (6) when the form (9) is used. With the modification as in Eq. (31) the correction will be $-1.2 \Gamma_{i}$ - very close to the numbers in Eqs. (56) and (57) found by Dewitt et al. (1973) by two different methods. See the more detailed discussion of this difference in Glazyrin \& Blinnikov (2010).

The difference between Eqs. (9) and (31) is explained by the neglect of electro-neutrality in the standard Eq. (9): the latter assumes independence of $n_{\mathrm{e}}$ and $n_{i}$, which is not true in general (Glazyrin \& Blinnikov 2010).

The argument on the role of the chemical potentials and on how to approach them still persists. For example, Brown et al. (2006) discuss the difference of their use of chemical potentials with that of Dewitt et al. (1973). Essentially, Brown et al. (2006) found that the Coulomb correction justified by Dewitt et al. (1973) for a classical plasma is applicable to a quantum plasma as well (see also Brydges \& Martin 1999, for a detailed review on the modern perspective on Coulomb systems).

We cannot use Eqs. $(29,31)$ directly in a multi-component plasma because $n_{\mathrm{e}}$ depends on the sum of many ions, nevertheless, those expressions should be meaningful in many cases when the distribution of nuclei is dominated by a set of species with similar values of $\left(A_{i}, Z_{i}\right)$. We compare the effect of changing corrections as in formula (9) and in (31) on the proton fraction in Fig. 15. The effect is visible only at highest density, but it is 

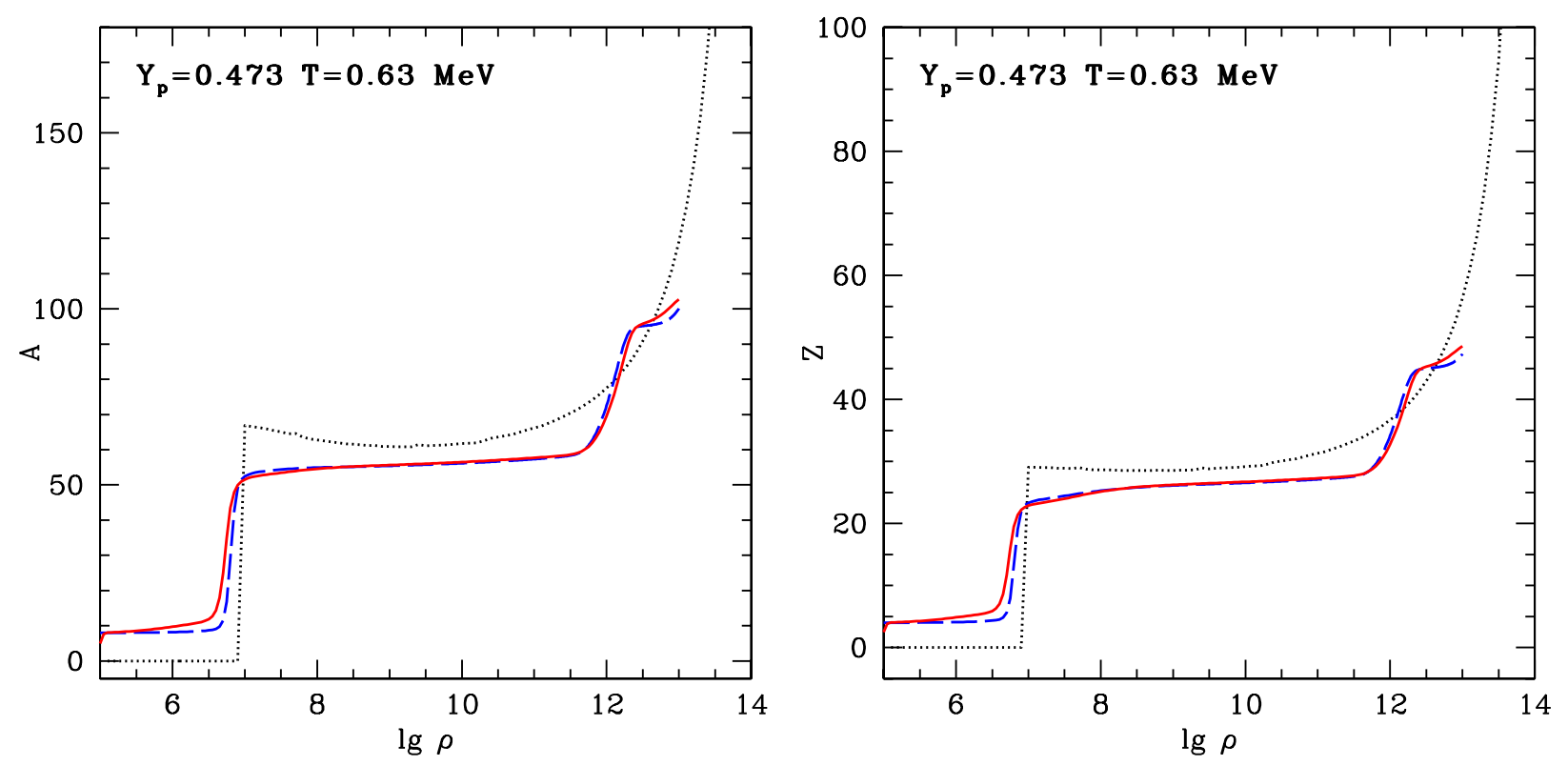

Fig. 9. Average mass number, $A$, (left) and average proton number, $Z$, (right) of nuclei as a function of density for $Y_{\mathrm{e}}=0.473$ and $T=0.63 \mathrm{MeV}$. The notation is the same as in Fig. 5.

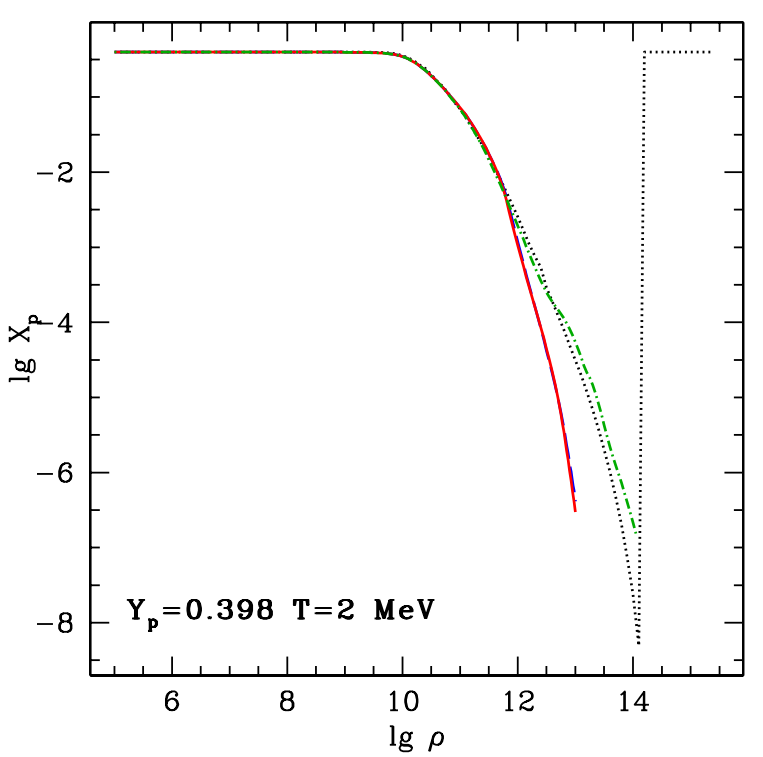

Fig. 10. Mass fraction of free protons, $X_{\mathrm{p}}$, as a function of density for $Y_{\mathrm{e}}=0.398$ and $T=2 \mathrm{MeV}$. The notation is the same as in Fig. 5 .

still very small. An effect slightly more pronounced is obvious in Fig. 16 for the average mass of heavy nuclei again at highest density when the NSE model is near the border of applicability. A more detailed study is needed here along the lines undertaken recently by Nadyozhin \& Yudin (2005).

\section{Summary and discussions}

We have studied the properties of hot and dense matter at the conditions relevant to supernova cores in the extended Saha approach. The multi-composition of nuclei is taken into account by solving the chemical equilibrium among nuclei, neutrons, and protons. The contributions to chemical potentials owing to the degeneracy of nucleons, to Coulomb, nuclear, and surface effects are included in the Saha formulation. The modification of binding energies of nuclei through the existence of nucleons outside nucleus is solved self-consistently. This is different from the treatment of Mazurek et al. (1979). We refer the reader to Nadyozhin \& Yudin (2005), where the difficulty of selfconsistent treatment is pointed out. We have adopted the recent mass formula by Koura et al. (2005), covering 20 000 nuclides, and compared the results with the ones by Hilf et al. (1976).

We have calculated extensive grids of density and temperature for several choices of $Y_{\mathrm{e}}$. We found that the obtained quantities behave in general very smoothly. When we compared our results with the values in the Shen EOS, which is a popular set of EOS for supernova simulations, they coincide closely for thermodynamical quantities. This may suggest that the one-species treatment of nuclei adopted in most of modern supernova EOS's is a good approximation in this regard. However, the composition of hot and dense matter in the extended Saha approach is broader than just a single heavy nucleus and alpha particles in the one-species approximation as in the Shen EOS. The appearance of nuclei starts with a mixture of various light nuclei at low density in the current approach. In the Shen EOS, ${ }^{4} \mathrm{He}$ is dominant at the same region and one-species nuclei appear at higher density. The average mass and proton numbers of nuclei (apart from ${ }^{4} \mathrm{He}$ ) are larger at low density and smaller at high density than those obtained from the Shen EOS. The former arises from the appearance of light nuclei around $A \sim 10$ in addition to alpha particles, which are only included in Shen EOS. We also explored the composition in the supernova core in the nuclear chart. We found that the distribution extends widely for high density and temperature and it depends on the choice of the mass formula, which provides different shell effects.

Our approach is fairly simple and may be compared with the more sophisticated modern treatment of nuclear reactions based on the statistical multi-fragmentation model (SMM): see Botvina \& Mishustin (2010). Recently the difference between the treatments under single and multi nuclear species approximations with phenomenological mass formulae has been explored 

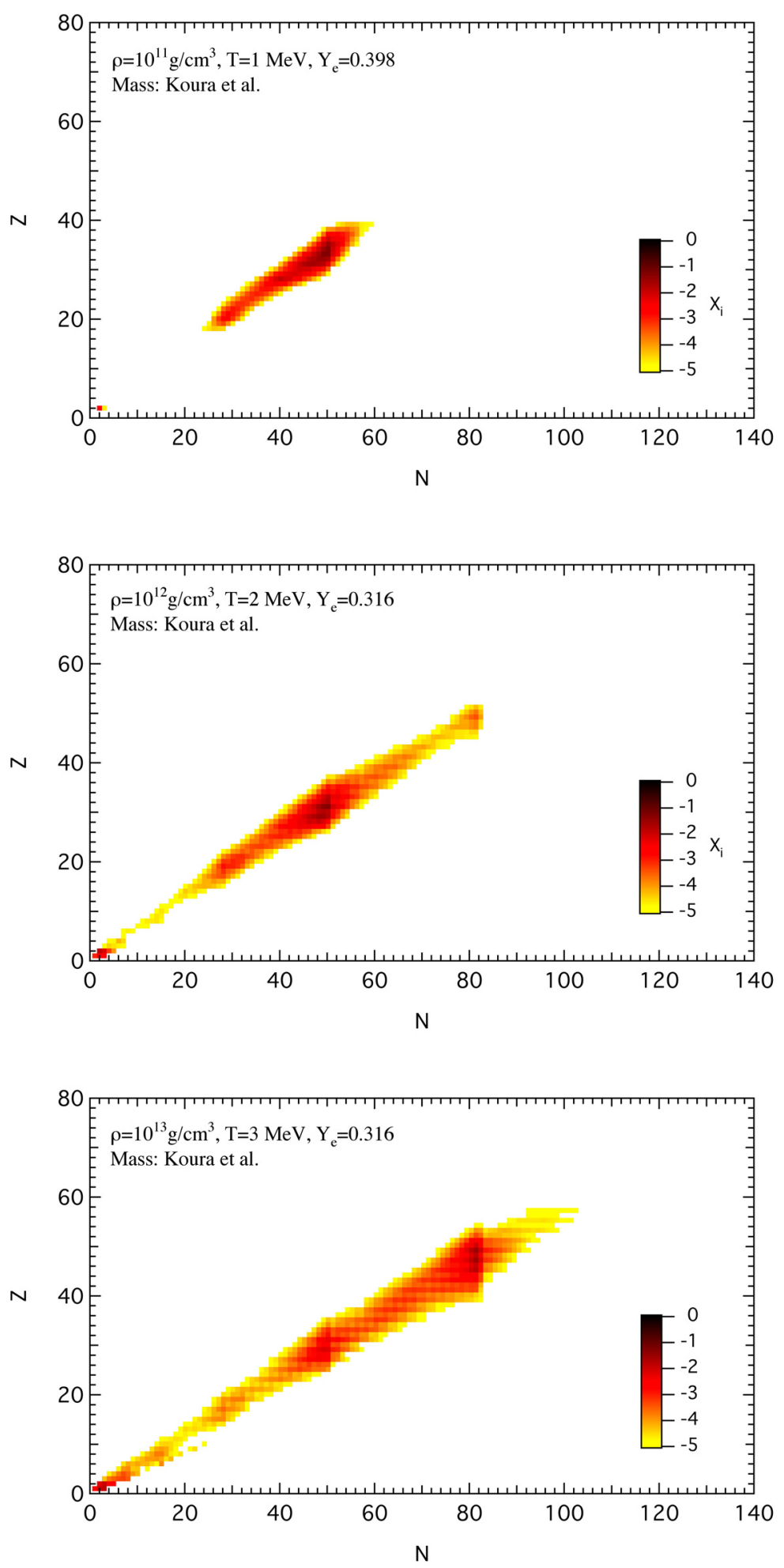

Fig. 11. Mass fraction of nuclei, $X_{A}$, with the proton number, $Z$, and neutron number, $N=A-Z$ in the nuclear chart for $\rho=10^{11} \mathrm{~g} / \mathrm{cm}^{3}, Y_{\mathrm{e}}=0.398$ and $T=1 \mathrm{MeV}$.
Fig. 12. Mass fraction of nuclei, $X_{A}$, with the proton number, $Z$, and neutron number, $N=A-Z$ in the nuclear chart for $\rho=10^{12} \mathrm{~g} / \mathrm{cm}^{3}, Y_{\mathrm{e}}=0.316$ and $T=2 \mathrm{MeV}$.
Fig. 13. Mass fraction of nuclei, $X_{A}$, with the proton number, $Z$, and neutron number, $N=A-Z$ in the nuclear chart for $\rho=10^{13} \mathrm{~g} / \mathrm{cm}^{3}, Y_{\mathrm{e}}=0.316$ and $T=3 \mathrm{MeV}$. to demonstrate the overestimation of the mass number in the single nuclear species by Souza et al. (2009).

Our results assist the understanding of the gravitational collapse of massive stars for supernova explosions. The difference of composition from the one-species treatment in the Shen EOS suggests that the proper treatment for a multi-composition of nuclei is important and may significantly affect the dynamics of core collapse. Because the electron capture on nuclei and free protons is crucial to determine the size of bounce core, i.e. the location of initial launch of shock wave, one should take the composition properly into account. These efforts have been made to evaluate the total electron capture rates on nuclei by averaging the distributions (Hix et al. 2003). One should, however, work carefully to predict the composition, because the mass and the nuclear effects may affect the detailed abundance. It is also necessary to calculate the equation of state as well as the thermodynamical quantities and compositions in a consistent manner. 


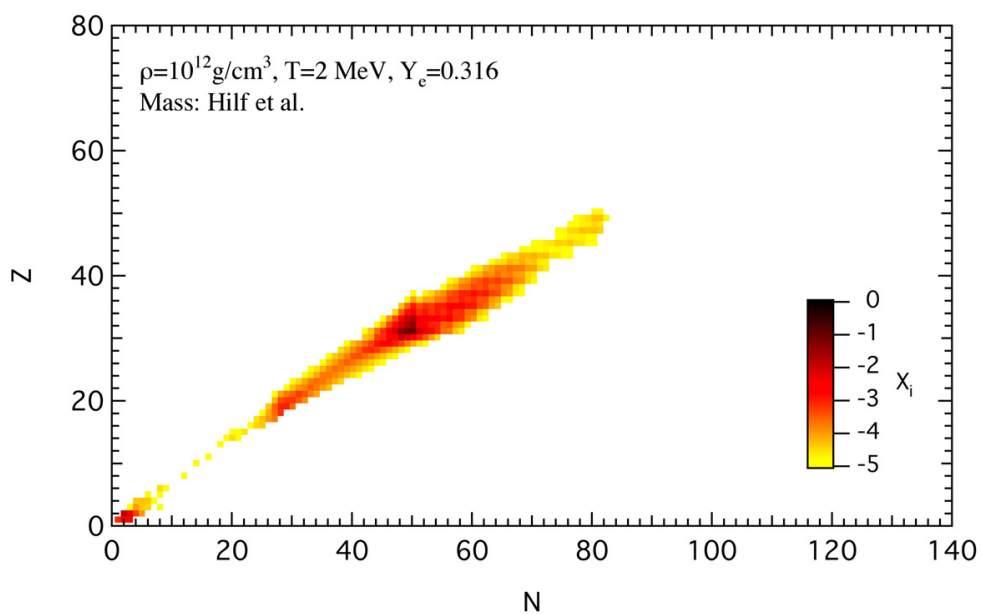

Fig. 14. Same as Fig. 12 but for Hilf et al. (1976).
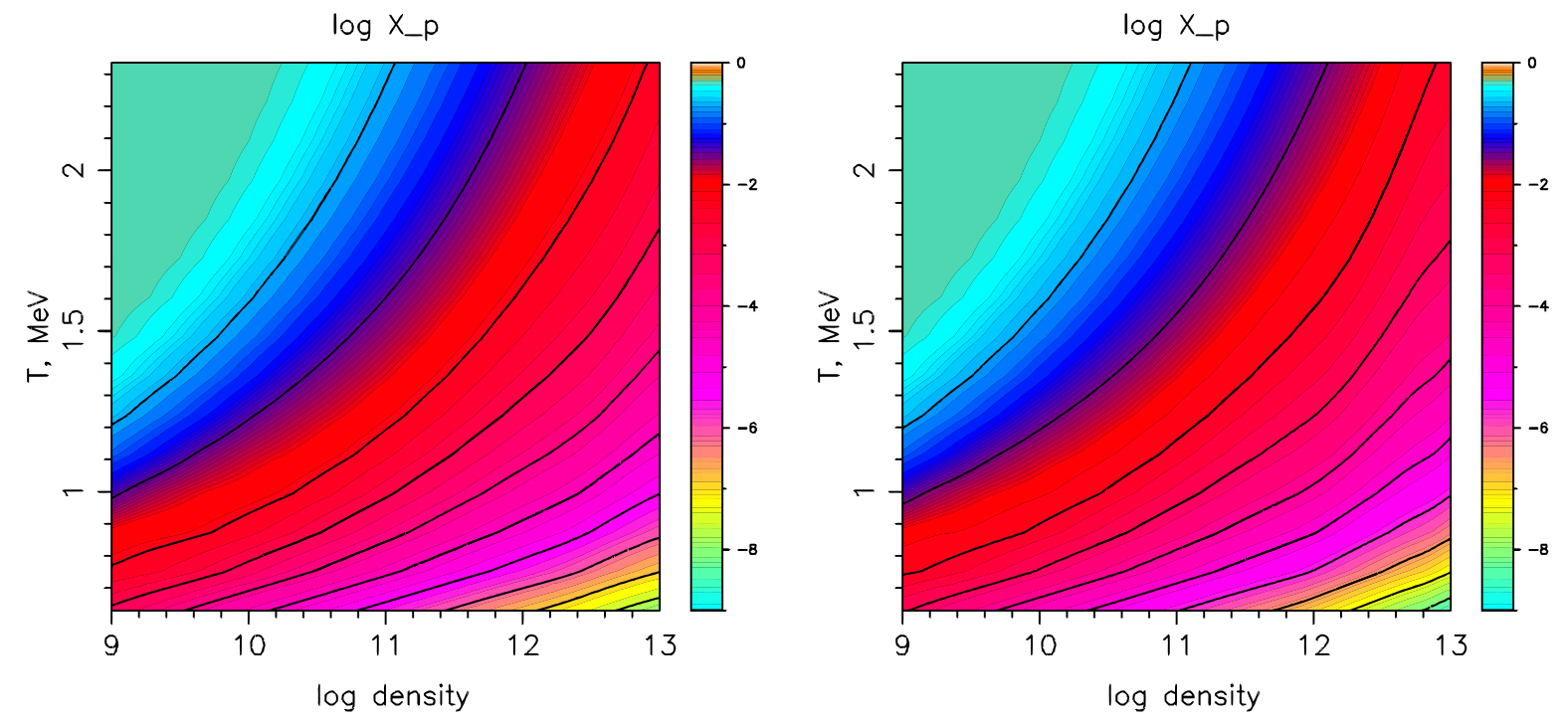

Fig. 15. Isocontours of the proton fraction, $X_{\mathrm{p}}$, for $Y_{\mathrm{e}}=0.473$. Left panel: the Coulomb correction is taken as in Eq. (8). Right panel: as in Eq. (31).
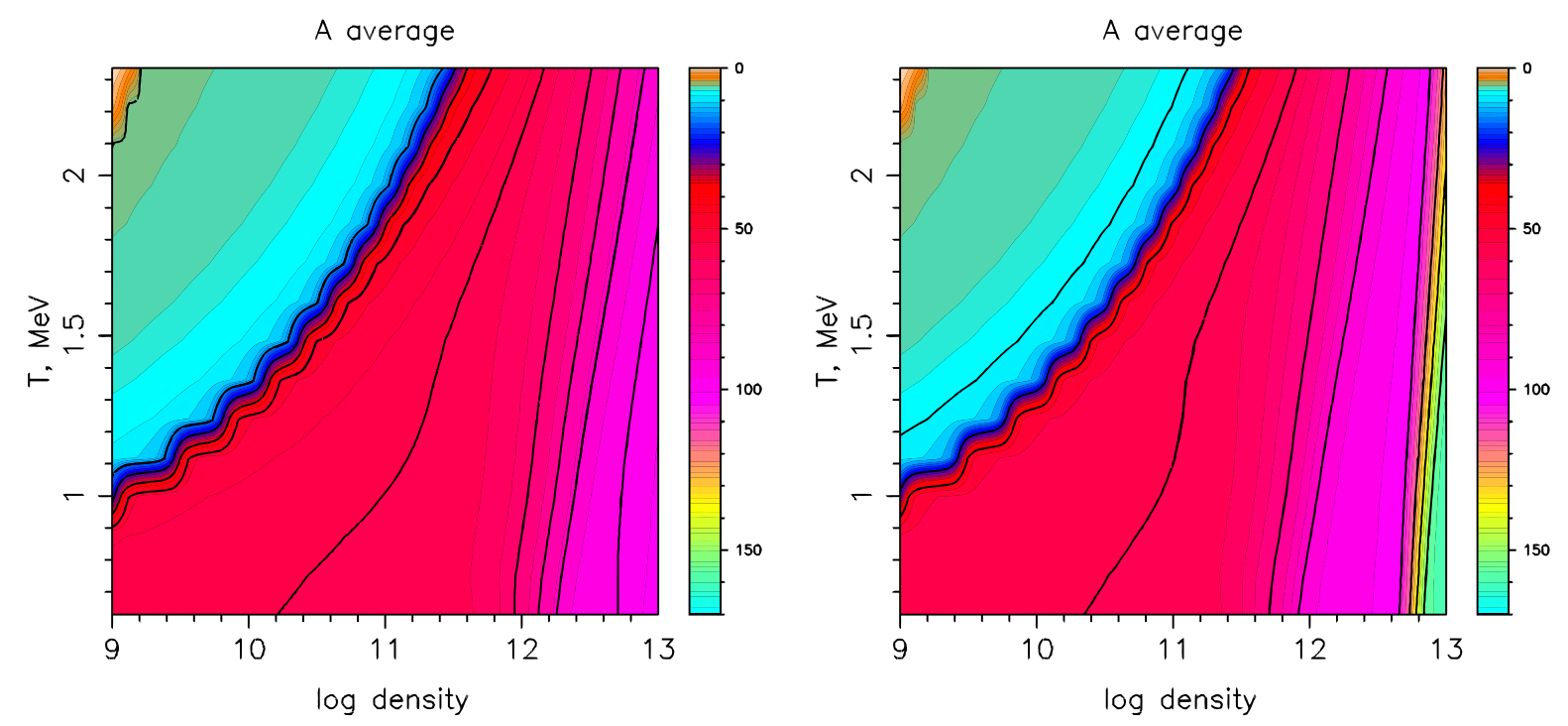

Fig. 16. Isocontours of the average mass number, $A$, of heavy nuclei, for $Y_{\mathrm{e}}=0.473$. Left panel: the Coulomb correction is taken as in Eq. (8). Right panel: as in Eq. (31). 
We are aiming to apply the extended Saha treatment to provide EOS sets for supernova simulations in the future.

Acknowledgements. A part of this work was made while S. Blinnikov, I. Panov, and K. Sumiyoshi were visiting the Max-Planck-Institut für Astrophysik, Garching. These authors are especially thankful to W. Hillebrandt, E. Müller, and H.-Th. Janka for the hospitality and to W.H. and E.M. for reading the manuscript at the early and final stages of the work. S.B. cordially thanks K. Nomoto, K. Sato, and H. Murayama for hospitality at RESCEU and IPMU in Tokyo, where he was able to complete the paper. S.B.'s work is supported by World Premier International Research Center Initiative (WPI), MEXT, Japan. We are grateful to D. Yakovlev for drawing our attention to the problem of Coulomb corrections in nuclear interactions and for his valuable consultations. The late C. Engelbrecht has given us his results on partition functions prior to publication. The work of S.B. and I.P. in Russia is partly supported by the grants RFBR 10-02-00249, 10-02-01398, by "Scientific School" grants 3458.2010.2, 3899.2010.2, by contract with Agency for Science and Innovation No. 02.740.11.0250, SNSF grant No. IZ73Z0-128180/1 under the programme SCOPES.

K.S. would like to thank C. Ishizuka, S. Furusawa, and H. Shen for helpful discussions. K.S. is partly supported by the Grants-in-Aid for the Scientific Research $(18540291,18540295,19104006,19540252,22540296)$ and by the Grants-in-Aid for Scientific Research on Innovative Areas of the MEXT (20105004) in Japan. S.B. is grateful to Elena Blinnikova for typing many long formulae in $\mathrm{T}_{\mathrm{E}} \mathrm{X}$, and to Mona Frommert for her valuable help at one of the critical stages of the project.

\section{References}

Arcones, A., Martínez-Pinedo, G., O’Connor, E., et al. 2008, Phys. Rev. C, 78, 015806

Arnould, M., Goriely, S., \& Takahashi, K. 2007, Phys. Rep., 450, 97

Baym, G., Bethe, H. A., \& Pethick, C. J. 1971, Nucl. Phys. A, 175, 225

Bethe, H. A. 1990, Rev. Mod. Phys., 62, 801

Bethe, H. A., Borner, G., \& Sato, K. 1970, A\&A, 7, 279

Blinnikov, S. I. 1987, Sov. Astron. Lett., C13, 346

Blinnikov, S. I., \& Rudzsky, M. A. 1988, Astrofizika, 29, 385

Blinnikov, S. I., Dunina-Barkovskaya, N. V., \& Nadyozhin, D. K. 1996, ApJS, 106, 171

Bonche, P., \& Vautherin, D. 1981, Nucl. Phys. A, 372, 496

Botvina, A., \& Mishustin, I. 2010, Nucl. Phys. A, 843, 98

Bravo, E., \& García-Senz, D. 1999, MNRAS, 307, 984

Brockmann, R., \& Machleidt, R. 1990, Phys. Rev. C, 42, 1965

Brown, L. S., Dooling, D. C., \& Preston, D. L. 2006, Phys. Rev. E, 73, 056406

Bruenn, S. W. 1989a, ApJ, 340, 955

Bruenn, S. W. 1989b, ApJ, 341, 385

Brydges, D. C., \& Martin, P. A. 1999, J. Stat. Phys., 96, 1163

Burrows, A., \& Lattimer, J. M. 1984, ApJ, 285, 294

Burrows, A., Livne, E., Dessart, L., Ott, C. D., \& Murphy, J. 2006, ApJ, 640, 878

Chabrier, G., \& Potekhin, A. Y. 1998, Phys. Rev. E, 58, 4941

Clifford, F. E., \& Tayler, R. J. 1965, MNRAS, 69, 21

Cooperstein, J. 1985, Nucl. Phys., A429, 527

Cooperstein, J., \& Baron, E. A. 1990, in Supernovae, ed. A. Petschek (New York: Springer-Verlag), 213

Dewitt, H. E., Graboske, H. C., \& Cooper, M. S. 1973, ApJ, 181, 439

El Eid, M. F., \& Hilf, E. R. 1977, A\&A, 57, 243

El Eid, M. F., \& Hillebrandt, W. 1980, A\&AS, 42, 215

Engelbrecht, C. A., \& Engelbrecht, J. R. 1991, Ann. Phys., 207, 1

Engelbrecht, C. A., Engelbrecht, J. R., \& Joubert, D. P. 1990, unpublished

Fowler, W. A., Engelbrecht, C. A., \& Woosley, S. E. 1978, ApJ, 226, 984

Furusawa, S., Yamada, S., Sumiyoshi, K., \& Suzuki, H. 2011, ApJ, 738, 178

Glazyrin, S. I., \& Blinnikov, S. I. 2010, J. Phys. A Math. Gen., 43, 075501

Haensel, P., Potekhin, A. Y., \& Yakovlev, D. G. 2007, Neutron stars, 1 Equation of state and structure, Astrophysics and Space Science Library (New York: Springer-Verlag), XXIII, 619

Hempel, M., \& Schaffner-Bielich, J. 2010, Nucl. Phys. A, 837, 210

Hilf, E. R., von Groote, H., \& Takahashi, K. 1976, in 3rd International Conference on Nuclei Far from Stability, Cargese, France, 19-26 May, CERN No. 76-13, 142

Hillebrandt, W. 1991, in High-Pressure Equations of State: Theory and Applications, ed. S. Eliezer, \& R. A. Ricci (Amsterdam: North-Holland), 399
Hillebrandt, W., \& Müller, E. 1981, A\&A, 103, 147

Hillebrandt, W., \& Wolf, R. G. 1985, in Nucleosynthesis: Challenges and New Developments, ed. W. D. Arnett, \& J. W. Truran (Chicago: University of Chicago Press), 131

Hix, W. R., Messer, O. E. B., Mezzacappa, A., et al. 2003, Phys. Rev. Lett., 91, 201102

Ishizuka, C., Ohnishi, A., \& Sumiyoshi, K. 2003, Nucl. Phys. A, 723, 517

Janka, H.-T., Buras, R., Kitaura Joyanes, F. S., et al. 2005, Nucl. Phys. A, 758, 19

Janka, H.-T., Langanke, K., Marek, A., Martinez-Pinedo, G., \& Müller, B. 2007, Phys. Rep., 442, 38

Koura, H., Uno, M., Tachibana, T., \& Yamada, M. 2000, Nucl. Phys. A, 674, 47

Koura, H., Tachibana, T., Uno, M., \& Yamada, M. 2005, Prog. Theor. Phys., 113, 305

Langanke, K., \& Martínez-Pinedo, G. 2003, Rev. Mod. Phys., 75, 819

Lassaut, M., Flocard, H., Bonche, P., Heenen, P. H., \& Suraud, E. 1987, A\&A, $183, \mathrm{~L} 3$

Lattimer, J. M., \& Swesty, F. D. 1991, Nucl. Phys., A535, 331

Lattimer, J. M., Pethick, C. J., Ravenhall, D. G., \& Lamb, D. Q. 1985, Nucl. Phys. A, 432, 646

Liu, M.-Q., Zhang, J., \& Luo, Z.-Q. 2007, Chin. Phys., 16, 3146

Mackie, F. D. 1976, Ph.D. Thesis, University of Illinois at Urbana-Champaign

Mazurek, T. J., Lattimer, J. M., \& Brown, G. E. 1979, ApJ, 229, 713

Möller, P., Nix, J. R., \& Swiatecki, W. J. 1995, Atomic Data Nucl. Data Tables, 59,185

Murphy, M. J. 1980, APJS, 42, 385

Nadyozhin, D. K. 1974a, Nauchnye informatsii, 32, 3, in Russian

Nadyozhin, D. K. 1974b, Nauchnye informatsii, 33, 117, in Russian

Nadyozhin, D. K., \& Yudin, A. V. 2004, Astron. Lett., 30, 634

Nadyozhin, D. K., \& Yudin, A. V. 2005, Astron. Lett., 31, 271

Nadyozhin, D. K., Panov, I. V., \& Blinnikov, S. I. 1998, A\&A, 335, 207

Nakamura, S. X., Sumiyoshi, K., \& Sato, T. 2009, Phys. Rev. C, 80, 035802

Nordheim, L. W. 1950, Phys. Rev., 78, 294

O’Connor, E., Gazit, D., Horowitz, C. J., Schwenk, A., \& Barnea, N. 2007, Phys. Rev. C, 75, 055803

Odrzywolek, A. 2009, Phys. Rev. C, 80, 045801

Odrzywolek, A. 2010 [arXiv: 1008.0798]

Panov, I., Blinnikov, S., \& Thielemann, F.-K. 2001, Astron. Lett., 27, 239

Ptitsyn, D. A., \& Chechetkin, V. M. 1982, Soviet Astron. Lett., 8, 322

Raduta, A. R., \& Gulminelli, F. 2010, Phys. Rev. C, 82, 065801

Rauscher, T. 2003, ApJS, 147, 403

Rauscher, T., \& Thielemann, F.-K. 2000, Atomic Data Nucl. Data Tables, 75, 1

Rauscher, T., Thielemann, F.-K., \& Kratz, K.-L. 1997, Phys. Rev. C, 56, 1613

Röpke, G., Münchow, L., \& Schulz, H. 1982, Nucl. Phys. A, 379, 536

Röpke, G., Schmidt, M., Münchow, L., \& Schulz, H. 1983, Nucl. Phys. A, 399, 587

Rosswog, S., \& Liebendörfer, M. 2003, MNRAS, 342, 673

Rüster, S. B., Hempel, M., \& Schaffner-Bielich, J. 2006, Phys. Rev. C, 73, 035804

Serot, B. D., \& Walecka, J. D. 1986, in Advances in Nuclear Physics, ed. J. W. Negele, \& E. Vogt (New York: Plenum Press), 16, 1

Shen, H., Toki, H., Oyamatsu, K., \& Sumiyoshi, K. 1998a, Nucl. Phys. A, 637, 435

Shen, H., Toki, H., Oyamatsu, K., \& Sumiyoshi, K. 1998b, Prog. Thoer. Phys., 100,1013

Souza, S. R., Steiner, A. W., Lynch, W. G., Donangelo, R., \& Famiano, M. A. 2009, ApJ, 707, 1495

Sugahara, Y., \& Toki, H. 1994, Nucl. Phys. A, 579, 557

Sumiyoshi, K., \& Röpke, G. 2008, Phys. Rev. C, 77, 055804

Sumiyoshi, K., Suzuki, H., Yamada, S., \& Toki, H. 2004, Nucl. Phys. A, 730, 227

Sumiyoshi, K., Yamada, S., Suzuki, H., et al. 2005, ApJ, 629, 922

Sumiyoshi, K., Yamada, S., Suzuki, H., \& Chiba, S. 2006, Phys. Rev. Lett., 97, 091101

Sutaria, F. K., Ray, A., Sheikh, J. A., \& Ring, P. 1999, A\&A, 349, 135

Suzuki, H. 1994, in Physics and Astrophysics of Neutrinos, ed. M. Fukugita, \& A. Suzuki (Tokyo: Springer-Verlag), 763

Swesty, F. D., Lattimer, J. M., \& Myra, E. S. 1994, ApJ, 425, 195

Typel, S., Röpke, G., Klähn, T., Blaschke, D., \& Wolter, H. H. 2010, Phys. Rev. C, 81,015803

Wolff, R. G. 1983, Ph.D. Thesis, Technische Universität, München

Woosley, S. E., \& Hoffman, R. 1992, ApJ, 395, 202

Yakovlev, D. G., \& Shalybkov, D. A. 1989, Astrophys. Space Phys. Rev., Soviet Sci. Rev. E., 7, 311 